\title{
Die großen wissenschaftlichen Leistungen von STefan Vogel (1925-2015) Teil 5b. Öl statt Nektar - die Ölblumen (dikotyle Vertreter)
}

\author{
Anton Weber, Günter Gerlach \& Stefan Dötterl
}

\begin{abstract}
In the course of evolution, oil flowers originated in 11 plant families, and even within these families oil flowers often evolved in parallel in several subgroups or genera. Based on the morphological analyses and pollination observations of STEFAN Vogel, in the present article significant representatives out of the dicotyledons are treated. With one exception, all families are of tropical or subtropical provenience. The exception are Primulaceae, in which about $40 \%$ of the species in the genus Lysimachia offer fatty oil as a floral reward. Oil-offering species of Lysimachia exhibit a holarctic distribution, with a few species occurring also in Central Europe. Pollination is by bees of the highly specialised genus Macropis (Melittidae).
\end{abstract}

\section{Zusammenfassung}

Ölblumen haben sich im Laufe der Evolution in 11 Pflanzenfamilien entwickelt und selbst innerhalb dieser Familien sind sie oft mehrfach und voneinander unabhängig entstanden. Im vorliegenden Artikel werden, aufbauend auf den morphologischen Analysen und Bestäubungsbeobachtungen von Stefan Vogel, wichtige Vertreter der Dikotyledonen vorgestellt. Die Familien sind meist tropisch- und subtropischer Provenienz. Eine Ausnahme bilden die Primulaceae, in der etwa 40 \% der Arten der Gattung Lysimachia fettes Öl als florales Attraktans anbieten. Lysimachia bzw. ihre ölblütigen Vertreter haben eine holarktische Verbreitung und sind mit einigen wenigen Arten auch in Mitteleuropa vertreten. Die Bestäubung erfolgt durch hochspezialisierte Bienen, den sogenannten Schenkelbienen (Macropis, Melittidae).

\section{Einführung}

Im Folgenden werden in Kurzform alle Familien der Dikotyledonen behandelt, die zur Gänze oder partiell Ölblumen enthalten. Die Reihenfolge richtet sich grob nach der Chronologie der Entdeckung und Erforschung, nur Lysimachia, also jene Gattung, die auch mitteleuropäische Vertreter mit Ölblumen beinhaltet, wird an das Ende gestellt. Die Monokotyledonen werden in der nächsten Ausgabe dieser Zeitschrift behandelt.

\section{Plantaginaceae: Angelonia, Basistemon, Monopera, Monttea}

Angelonia-Arten, die im Botanischen Garten der Universität Mainz kultiviert wurden, bildeten den Ausgangspunkt für die Entdeckung und Erforschung der Ölblumen. Tatsächlich handelt es sich bei Angelonia um eine hochinteressante Gattung, zu der Vogel und seine Schüler immer wieder zurückgekehrt sind. Verschiedene Aspekte wurden in nicht weniger als 13 Publikationen und Tagungsbeiträgen behandelt (z. B. Vogel 1974, 2002, Vogel \& Machado 1991, Machado et al. 2002).

Die Gattung Angelonia umfasst etwa 30 Arten, die von Mexiko bis Argentinien verbreitet sind.
Die bekannteste Art ist $A$. angustifolia aus Brasilien, die bei uns oft als Zierpflanze in zahlreichen Farbvarianten gezogen wird.
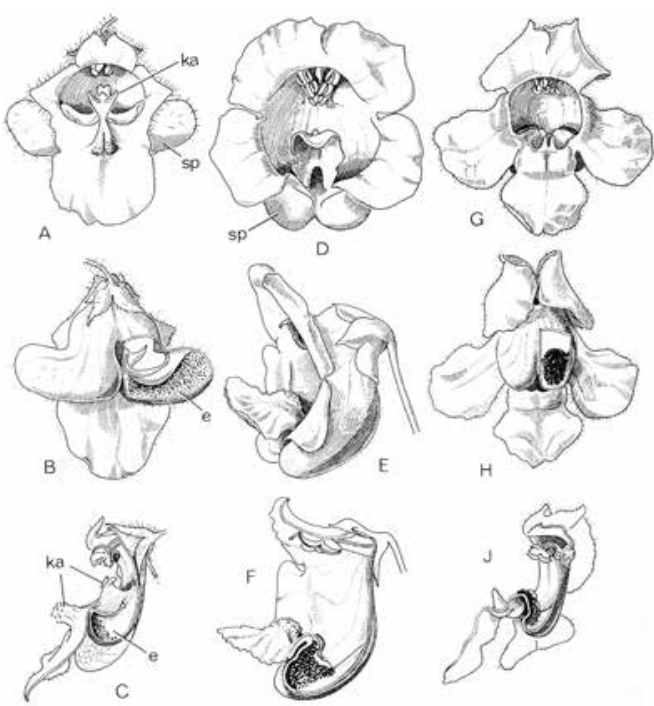

Abb. 1: Ölblumen der Gattung Angelonia (Plantaginaceae). A-C: A. pubescens, D-F: A. integerrima, G-J: A. angustifolia. Blüte jeweils in Vorder- und Rückenansicht sowie längs geschnitten, der sackartige Sporn in B, C, F, H und J aufgeschnitten und den Elaiophor zeigend; ka: Lippenkallus, sp: Sporn. (aus Vogel 1974, Abb. 10) 


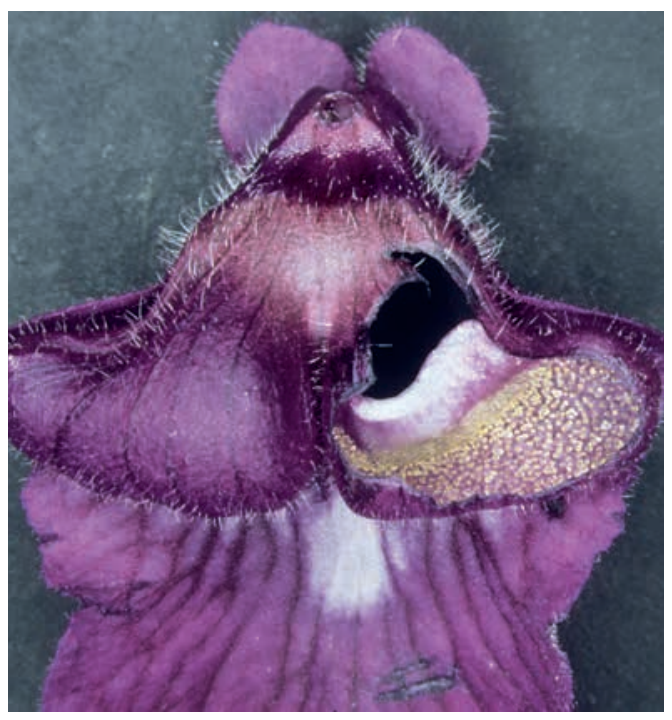

Abb. 2: Blüte von Angelonia pubescens (Plantaginaceae) in Rückenansicht; der rechte sackartige Sporn ist aufgeschnitten und zeigt den aus dicht gestellten Öldrüsen bestehenden Elaiophor (Trichom-Elaiophor). Vgl. dazu auch Abb. 1B. (Foto: G. Gerlach; Brasilien, Edo. Ceará, Fortaleza).

Die Blüte hat auf der Rückseite zwei sackartige Ausstülpungen, in der sich je ein grünlich gefärbter Trichom-Elaiophor befindet. Er liegt an der fast senkrecht gestellten Vorderwand der Ausstülpung und besteht aus dicht gestellten Drüsenhaaren mit länglichen, mehrzelligen Köpfchen.

Die Beobachtung der Bestäubung gelang Vogel erstmals am 11. September 1964 an Angelonia biflora (Vogel 1974). Er konnte zwei Bienenarten feststellen, die zur Gattung Centris gehören: C. trigonoides und C. fuscata. Wie Vogel mitteilt, näherten sie sich in rasantem Anflug den Blüten, landeten auf der Unterlippe, schlüpften zur Hälfte in den Schlund, schabten mit den Vorderbeinen das Öl aus den Ausstülpungen und führten in einem kurzen Schwebeflug Höselbewegungen durch.

Man muss nicht nach Südamerika reisen, um die Bestäubung von Angelonia zu sehen. Man kann dies bequem vor dem Bildschirm oder am Smartphone tun: Zusammen mit einem professionellen Filmteam drehte Vogel 1992 einen Dokumentarund Lehrfilm, der 2002 unter dem Namen „Bestäubung von Angelonia hirta (Scrophulariaceae)“

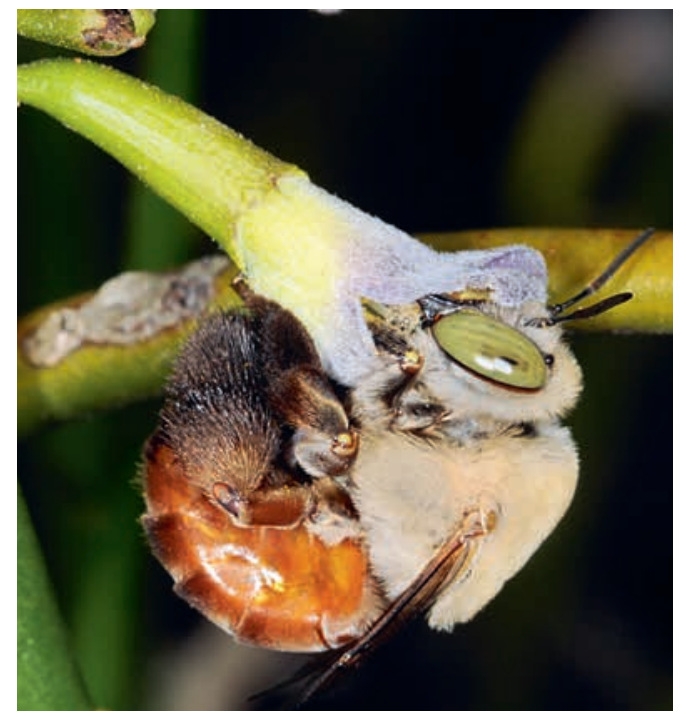

Abb. 3: Die Biene Centris brethesii (Apidae-Apinae-Centridini) beim Blütenbesuch von Monttea aphylla (Plantaginaceae). (Foto: A. A. Cocucci; Argentinien, Prov. La Rioja, Tambillos, Los Tambillos).

veröffentlicht wurde (Vogel 2002b). Er kann im Internet unter der Adresse https://av.tib.eu/ media/15808 abgerufen werden.

Neben Angelonia sind bei den Plantaginaceae noch drei weitere Gattungen mit Ölblumen gefunden worden: Basistemon (8 Arten), Monopera (2 Arten) und Monttea (3 Arten). An der Erforschung ihrer Ökologie und Bestäubung sind die beiden argentinischen Schüler Vogels, Alicia N. Sérsic und Andrea A. Cocucci, wesentlich beteiligt. Basistemon wurde eingehend von Vogel \& Cocucci (1995) studiert, wobei Paratetrapedia-Arten als Hauptbestäuber festgestellt werden konnten. Für Monopera liegt bisher nur die Beobachtung vor, dass Caenonomada bruneri als Bestäuber in Erscheinung tritt (Aguiar \& Melo 2009). Die Ölblütigkeit von Monttea wurde von Simpson et al. (1990) entdeckt, strukturelle und funktionelle Besonderheiten wurden später eingehend von SÉrSIC \& Cocucci (1999) studiert. TADEy (2011) konnte als Bestäuber von Monttea aphylla drei Bienenarten beobachten, Mesonychium jenseni, Centris brethesi und Centris vardyorum, wobei letztere auf Monttea spezialisiert ist. 


\section{Calceolariaceae: Calceolaria}

Die Pantoffelblumen sind wegen ihrer aparten, meist leuchtend gelben Blüten, deren Unterlippe schuhförmig aufgeblasen ist, beliebte und allgemein bekannte Zierpflanzen. Ihre Artenzahl wird meist mit etwa 250-270 angegeben, doch gibt es auch weitaus höhere Schätzwerte. Die Hauptverbreitung hat die Gattung in den Anden, nach Süden reicht sie bis zur Südspitze Südamerikas, nach Norden hin gibt es auch in Mittelamerika und im südlichen Mexiko noch zahlreiche Arten.

Der Bauplan der Blüte ist komplex und wurde lange Zeit missverstanden. Der Kelch ist vierzählig, die Krone 2-lippig (ohne weitere Gliederung), Staubblätter sind zwei vorhanden und der halbunterständige Fruchtknoten ist aus 2 Karpellen aufgebaut. Man hat den Blütenbau traditionell als Reduktionsform und Modifikation des 5-zähligen Bauplanes der Braunwurzgewächse (Scrophulariaceae) interpretiert und angenommen, dass von den 5 Kelchzipfeln die beiden unteren verwachsen seien, die beiden Lippen der Krone aus 2 bzw. 3 miteinander verwachsenen Blütenblättern bestünden, und die beiden Staubblätter Reste eines ursprünglich 5-zähligen Androeceums darstellen würden (wie das bei vielen Scrophulariaceen tatsächlich der Fall ist). Grund genug jedenfalls, Calceolaria und zwei weitere Gattungen, Jovellana und Porodittia (= Stemotria), zu den Scrophulariaceen zu stellen. Auf Grund molekular-phylogenetischer

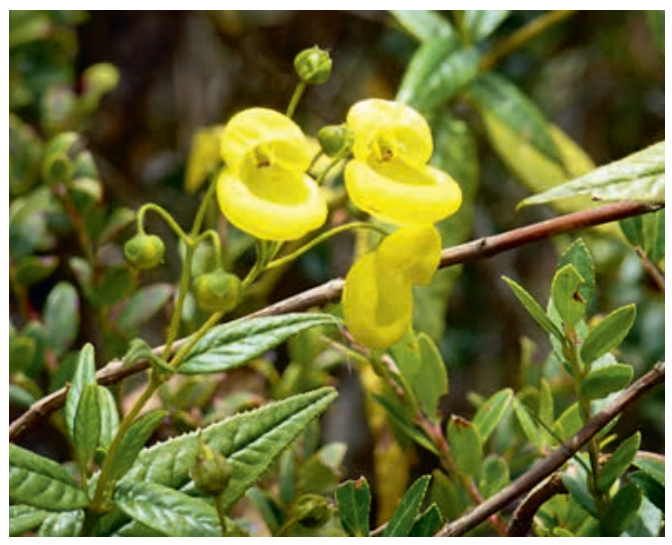

Abb. 4: In der etwa 270 Arten umfassenden Gattung Calceolaria (Calceolariaceae) bieten mehr als 200 Arten Öl als florales Attraktans an. Im Bild Calceolaria irazuensis. (Foto: A. Weber; Costa Rica, Torres Cerro de la Muerte)
Untersuchungen stellte sich jedoch heraus, dass die Calceolarien nichts mit den Scrophulariaceen zu tun haben und in eine eigene Familie, Calceolariaceae, gestellt werden müssen, die zwischen den Ölbaumgewächsen (Oleaceae) und den Gesneriaceen steht (Olmstead 2001). Später wurde Porodittia in Calceolaria eingegliedert (ANDERSSON 2006). Blütenentwicklungsgeschichtliche Untersuchungen zeigten, dass die Blüte aus Zweier-Wirteln aufgebaut ist und nicht von einem 5-zähligen Bauplan hergeleitet werden kann (MAYR \& WEBER 2006). Die Blüte ist in der Grundstruktur viel eher mit den Blüten der Ölbaumgewächse (Oleaceae) als mit den Scrophulariaceen in Beziehung zu setzen, was perfekt zu den molekularen Daten passt.

Schneidet man den Schuh einer Calceolaria-Blüte längs durch, sieht man, dass er nicht hohl bzw. leer ist (wie etwa beim Frauenschuh). Der apikale Teil der Unterlippe ist um- und in den Schuh hineingeschlagen. Bei vielen Arten trägt dieser Lappen einen einzigen, aus dicht gestellten Drüsen bestehenden Elaiophor auf der Unterseite. Dabei ist zu betonen, dass die topographische Unterseite nicht, wie bei Angelonia und Diascia, die Innenseite des betreffenden Kronblattabschnittes repräsentiert, sondern die morphologische Außenseite. Noch häufiger ist, dass der in den Schuh ragende Lappen stark verlängert und innerhalb des Schuhs nochmals umgeschlagen ist. Die beiden Lappen bilden ein Tasche oder Falte, und das ist der Ort, an dem der Elaiophor (wiederum an der morphologischen Außenseite) gelegen ist. Wie schon erwähnt, muss die Biene oft ein akrobatisches Kunststück vollführen, um sich in die Blüte hineinzuzwängen und das Öl mit den Beinen abzusammeln. Bei einigen Calceolaria-Arten ist ein Schlagbaum-Mechanismus nach Art des Wiesensalbeis vorhanden.

Anlässlich seiner Südamerika-Reise 1969/70 konnte Vogel von Anfang Dezember bis Ende Februar ein Dutzend Calceolaria-Arten am Standort (argentinische Kordillere) studieren und den Bestäubungsvorgang aufklären. Als Bestäuber konnte er Bienen der Gattungen Centris und Tapinotaspis beobachten und photographisch sowie zeichnerisch dokumentieren. 


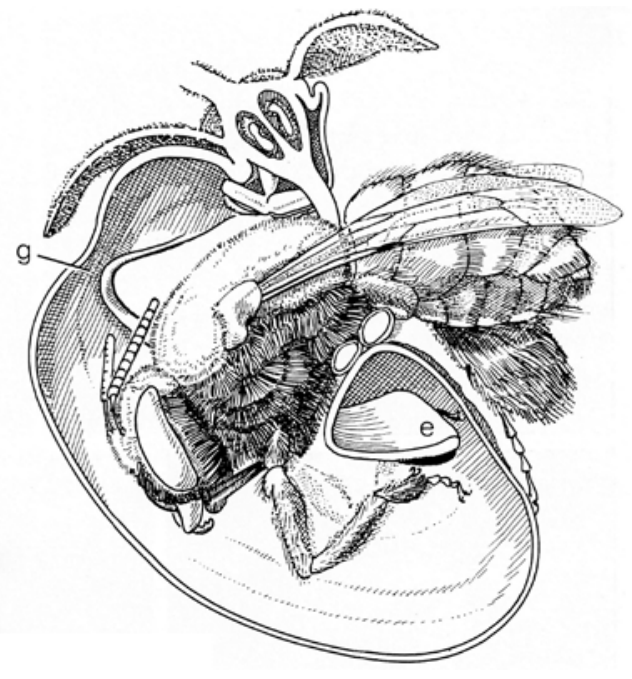

Abb. 5: Blüte von Calceolaria schickendantziana (längs aufgeschnitten) mit einer in die Blüte eindringenden Biene (Centris autrani). Die Biene sammelt mit den Vorderbeinen das Öl vom Elaiophor (e) ab, die Narbe schabt dabei den auf dem Rücken abgelagerten Fremdpollen ab. (aus VogeL 1974, Abb. 50B)

Heute sind wir durch die ausgedehnten Studien von Vogels argentinischer Schülerin Alicia SÉRSIC über die Bestäubung südamerikanischer Calceolarien sehr gut unterrichtet (SÉRSIC 2004).

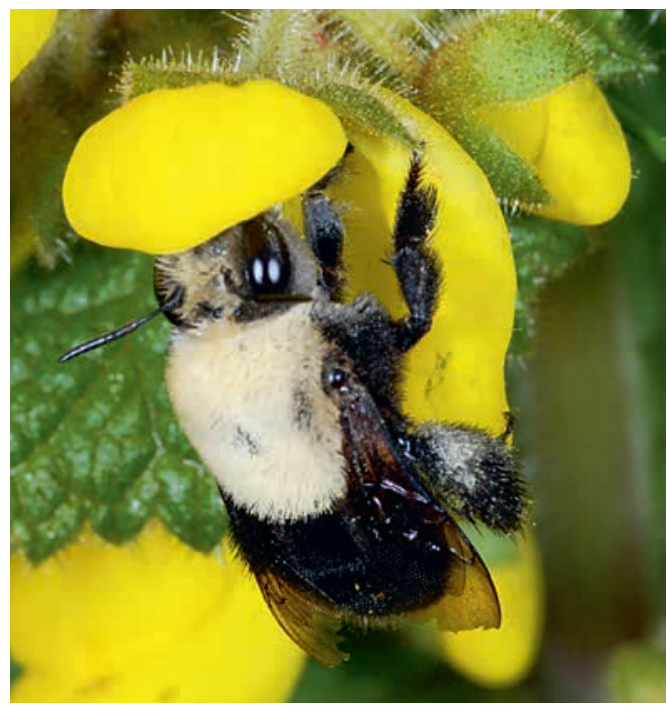

Abb. 6: Blüte von Calceolaria plectranthifolia mit der Biene Centris neffi (Apidae-Apinae-Centridini).

(Foto: A. A. Cocucci, Argentinien, Prov. Tucumán, nahe Tafí del Valle)
Sie konnte Vogels grundlegende Erkenntnisse an einer großen Zahl von Arten bestätigen, im Detail dokumentieren und beträchtlich erweitern.

Wichtig ist festzuhalten, dass nicht alle Calceolaria-Arten einen Elaiophor besitzen. Bei etwa einem Viertel der Arten fehlt ein solcher, ebenfalls bei allen Arten von Jovellana. Ihre Blüten stellen Pollenblumen dar oder sind zur Selbstbestäubung übergegangen.

Am Rande erwähnt sei, dass es noch eine weitere, höchst kuriose Ausnahme gibt: Calceolaria uniflora (= C. darwinii), aus den Bergen Patagoniens. Diese Art besitzt ockergelb-braune, rot-gesprenkelte Blüten, die außen auf dem Schuh einen schneeweißen, speckig-fleischigen Auswuchs tragen. Vogel (1974) deutete diesen als „Beköstigungsmittel“ (Futterkörper) und spricht von einem „sehr ungewöhnlichen Fall von Ornithophilie“. Dass diese geradezu abenteuerliche Interpretation voll zutrifft, wurde mehr als 20 Jahre später von SérsiC und CocuCCi bewiesen. Drosselartige, im Verhältnis zur Blüte riesige Vögel (Thinocorus rumicivorus, Zwerghöhenläufer), picken die weißen Futterkörper von den Blüten und übertragen dabei den Pollen von einer Blüte zur

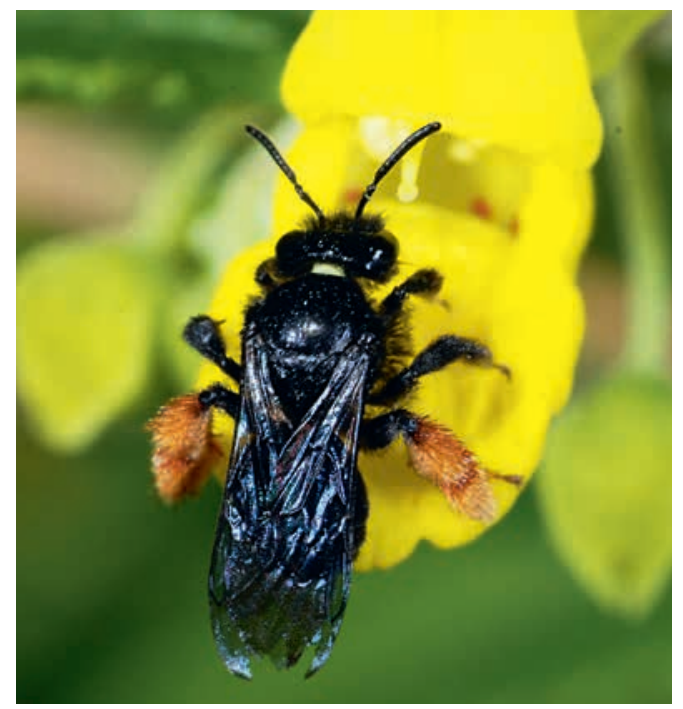

Abb. 7: Blüte von Calceolaria polyclada mit der Biene Chalepogenus rufipes (Apidae-Apinae-Tapinotaspidini). (Foto: A. A. Cocucci; Argentinien, Prov. Tucumán, nahe Tafí del Valle) 


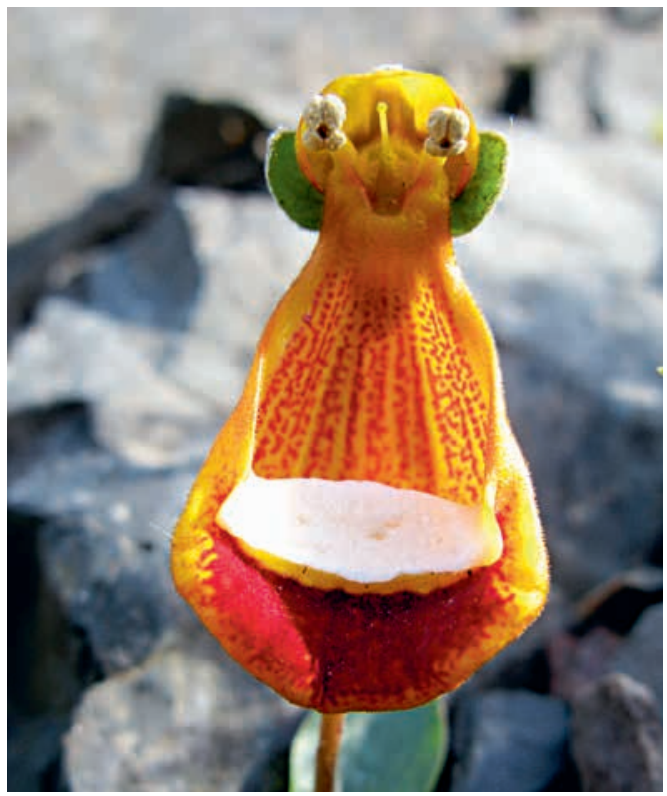

Abb. 8: Calceolaria uniflora ist weder eine Öl- noch eine Pollenblume, sondern wird von Vögeln (Zwerghöhenläufer, Thinocorus rumicivorus) bestäubt, welche den weißen, fleischigen Gewebekörper vom Pantoffel picken und dabei den Pollen übertragen. (Foto: Butterfly voyages, SERGE Ouachée, 2008; Chile, Torres del Paine. https://commons. wikimedia.org/wiki/File:Calceolaria_uniflora_Lam.jpg.; Lizenz: CC BY-SA 3.0)

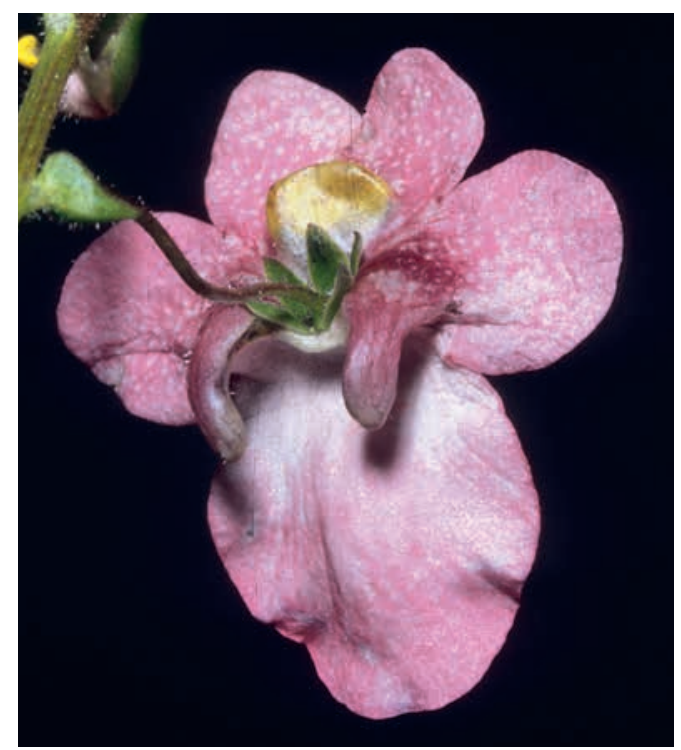

Abb. 9: Blüte von Diascia barberae (Scrophulariaceae) in Rückenansicht; die beiden ölführenden Sporne sind parallel nach hinten gerichtet. (Foto: G. Gerlach; cult. BG München-Nymphenburg) anderen. Ähnliches gilt wahrscheinlich auch für Calceolaria fothergilli, welche auf den Falkland-Inseln endemisch ist (SÉrsic \& CocuCCI 1996).

\section{Scrophulariaceae: Diascia}

Die Gattung Diascia ist mit etwa 70 Arten im südlichen Afrika verbreitet. Einige Arten (z. B. D. barberae), Hybriden und Kultivare haben Eingang in die Kultur gefunden. Die Blüten haben zwei nach hinten-unten gerichtete Hohlsporne und werden deshalb im Englischen „twinspurs“ („Zwillingssporne“) genannt. Auch der Deutsche Name, Elfensporn, nimmt auf die auffälligen Sporne Bezug. Die Arten unterscheiden sich unter anderem durch die Länge der Sporne. Es gibt praktisch ein Kontinuum von relativ kurzen $\mathrm{zu}$ ausgesprochen langen Spornen.

Wie Stefan Vogel bereits 1974 beschrieb, sind die Sporne innen mit ölabscheidenden Drüsen besetzt. Auf Grund dieser Tatsache machte er eine Reihe von Voraussagen über die damals noch völlig unbekannten Bestäuber und ihre Methode der Ausbeutung. Diese trafen in den 1980er Jahren sukzessive und punktgenau ein.

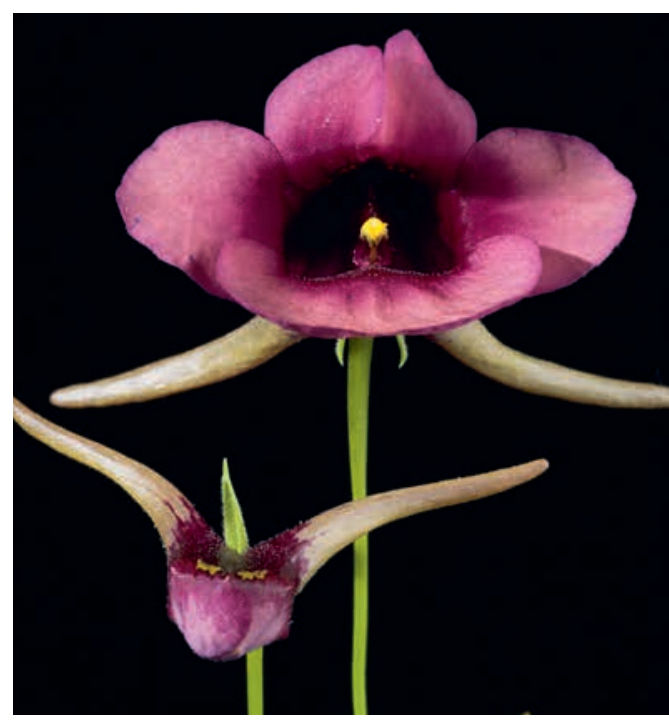

Abb. 10: Blüten und Blütenknospe von Diascia whiteheadii. Die langen, dünnen und auseinander gespreizten Sporne können nur von Rediviva-Arten mit extrem langen Vorderbeinen (besonders $R$. emdeorum) ausgebeutet werden. (Foto: A. PAuw; Süd-Afrika, West-Kap, Cederberg) 


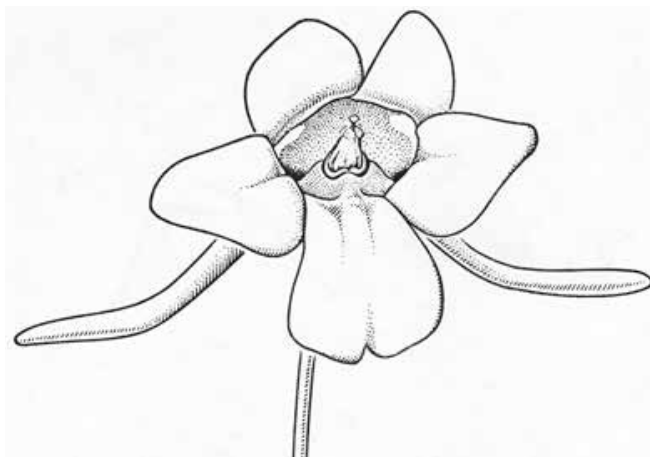

Abb. 11: Blüte von Diascia longicornis. (aus Vogel \& Michener 1985, Fig. 3; Zeichnung S. Vogel)

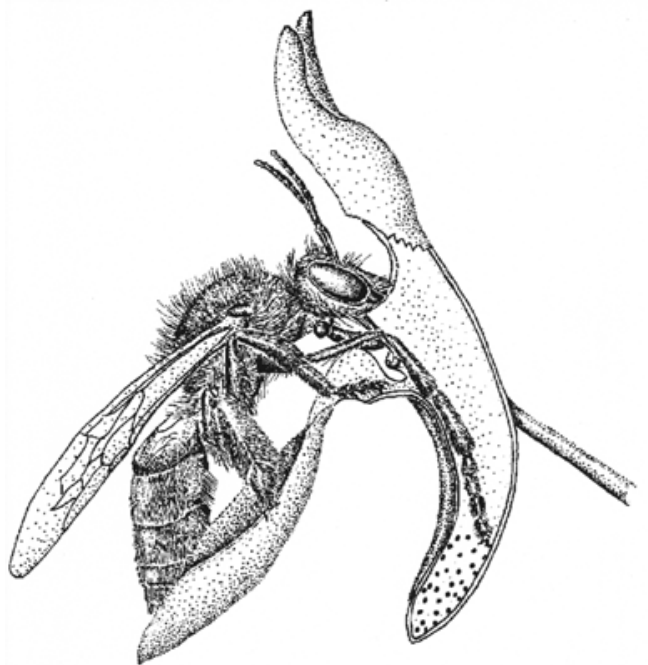

Abb. 12: Blüte in Seitenansicht und aufgeschnitten, mit ihrem Bestäuber Rediviva emdeorum; die Bienen greift mit ihren langen Vorderbeinen in die Sporne hinein um das Öl aufzusammeln. (aus Steiner \& Whitehead 1990, Fig. 1)

Was man heute weiß, lässt sich in kurzer Form folgendermaßen zusammenfassen (vgl. VogeL 1974, Steiner \& Whitehead 1988, 1990, 1991 u. a., PAuw et al. 2017):

(1) Die stets paarig angeordneten Sporne, die innen den Elaiophor tragen, werden durch Bienen ausgebeutet, welche die beiden Vorderbeine zum Ölsammeln benutzen („Zwei-Bein-Sammler“).

(2) Die dazu fähigen Bienen gehören alle der Gattung Rediviva (Melittidae-Mellitinae-Mellitini) an. Diese Gattung umfasst etwa 20 Arten, die alle auf

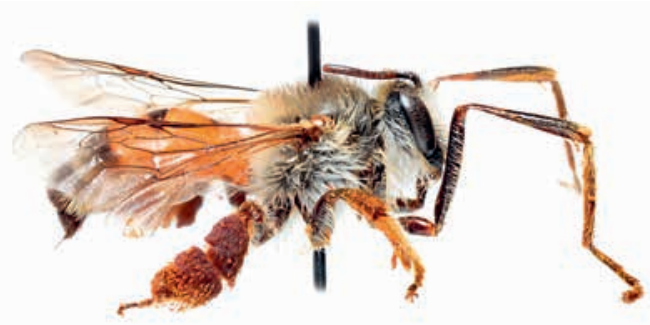

Abb. 13: Aufgespießtes Exemplar der Biene Rediviva emdeorum (Melittidae-Melittinae-Melittini).

(Foto: M. KuhlmanN)

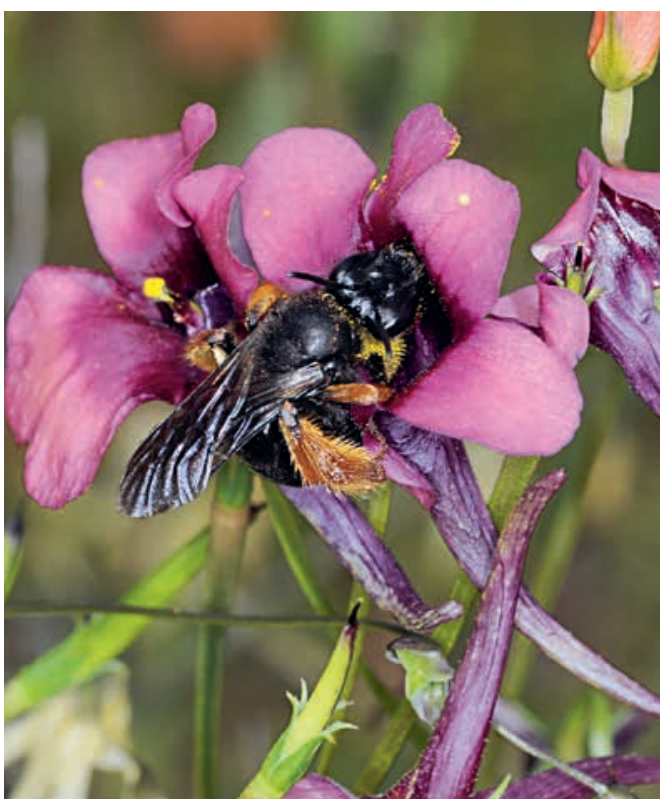

Abb. 14: Blüte von Diascia cf. longicornis mit ihrem Bestäuber Rediviva longimanus. (Foto: A. A. CocuccI; Süd-Afrika, Südwest-Kap, Clanwilliam)

das Ölsammeln spezialisiert sind. Die sieben Arten der Schwestergattung Redivivoides sind dazu nicht befähigt (KuHLmann 2012).

(3) Das Öl wird wie sonst nur von den Weibchen gesammelt und - mit Pollen vermischt - als Larvenproviant genutzt.

(4) Die Diascien stellen die Hauptquelle für die ölsammelnden Bienen Südafrikas dar, wenngleich auch in anderen Scrophulariaceen-Gattungen (Alonsoa, Colpias, Hemimeris; vgl. Steiner 1989, Whitehead \& Steiner 2001, Steiner \& 
Whitehead 2002) und weiteren Familien (siehe Iridaceae und Orchidacae im nächsten Beitrag dieser Serie) Ölblumen zur Verfügung stehen.

(5) Die Blüten von Diascia sect. Racemosae besitzen an der Basis der Oberlippe ein durchscheinendes Fenster, das gelb gefärbt und mit kastanienbraunen Flecken versehen ist. Es ist entweder flach oder kegelfömig vertieft. Das Fenster dient den ölsammelnden Bienen als visuelle Orientierungshilfe (Steiner 1990).

\section{Stilbaceae: Anastrabe, Bowkeria, Ixianthes}

Wie Diascia sind auch Bowkeria, Anastabe und Ixianthes in Südafrika beheimatet. Sie gehören der kleinen, 12 Gattungen und etwa 40 Arten umfassenden Familie der Stilbaceae an, die erst kürzlich von den Scrophulariaceen abgespalten wurde. Wie Vogel (1974) zunächst für Bowkeria verticillata feststellen konnte und für Anastrabe vermutete, besitzen die Blüten einen Trichom-Elaiophor. Anastrabe und Ixianthes (mit je einer Art) besitzen Rachenblüten, wie sie für die Lamiales typisch sind: mit einer zweiteiligen Oberlippe, einer (schwach bis ausgeprägt) 3-teiligen Unterlippe und einem offenem Schlund. Bei Bowkeria (5 Arten) lässt sich eine Progression von Rachen- zu Maskenblüten beobachten. So ist z. B. bei Bowkeria citrina die Blüte flachgedrückt und der Schlund quer verengt.

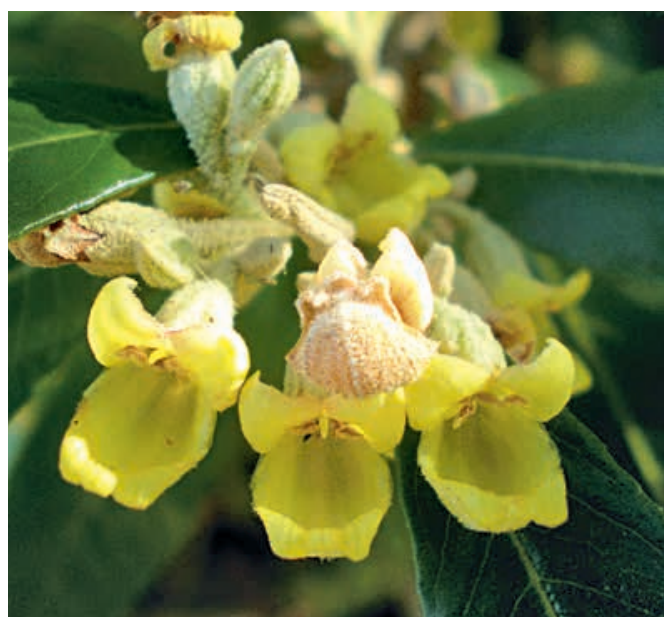

Abb. 15: Blüten von Anastrabe integerrima (Stilbaceae). (Foto: Consultaplantas, 23.01.2014, Jardín Botánico de Barcelona: https://de.wikipedia.org/wiki/Stilbaceae\#/media/ File:Anastrabe_integerrima_2c.JPG. Lizenz: CC BY 4.0)

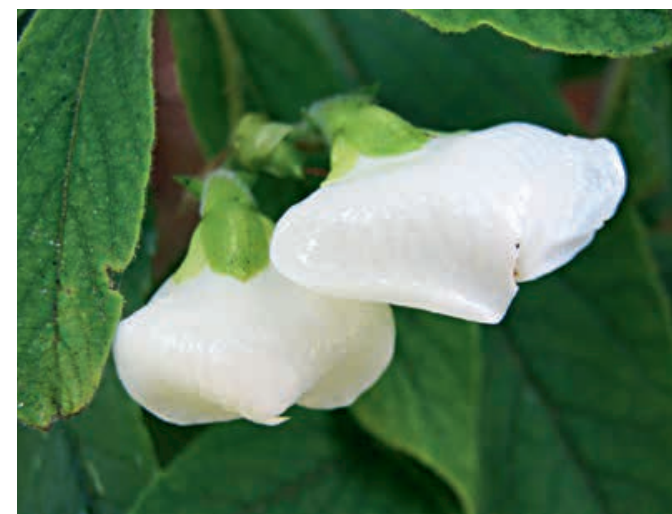

Abb. 16: Blüten von Bowkeria verticillata (Stilbaceae). Der Schlund wird durch die dachartige Oberlippe völlig verschlossen. (Foto: Melburnian; Geelong Botanic Gardens, Victoria, Australia; 3 Apr 2010; https://commons.wikimedia.org/wiki/File:Bowkeria_verticillata.jpg. Lizenz: CC BY 3.0.)

Bei der von Vogel untersuchten $B$. verticillata ist der Schlund hingegen völlig verschlossen, die Blüte stellt eine besondere Form einer Maskenblüte dar. Der Elaiophor besteht aus einem Drüsenfeld, welche das Innere der bauchig erweiterten Kronröhre fast völlig auskleidet.

Wir wissen heute durch die Untersuchungen von K. E. Steiner und V. B. Whitehead (Steiner \& Whitehead, 1990, 1991, 1996; Whitehead \& Steiner 1992; Steiner 1993), dass die ölblütigen Stilbaceen von Bienen der Gattung Rediviva besucht und bestäubt werden. Diese über 20 Arten umfassende Bienengattung kennen wir bereits von Diascia und sie ist auch für die Bestäubung anderer südafrikanischer Ölblumen, insbesondere aus den Orchideen, relevant.

\section{Solanaceae: Nierembergia}

Diese mit den Petunien verwandte Gattung ist bisher die einzige der über 100 Gattungen und etwa 2500 Arten umfassenden Familie der Nachtschattengewächse (Solanaceae), welche Ölblumen besitzt. Sie umfasst 21 Arten, deren Verbreitungsgebiet sich von Mexico bis in das südliche Südamerika erstreckt. Das Öl wird von einem Trichom-Elaiophor abgesondert, der an der Basis des Krontubus gelegen ist und sich auch auf die miteinander postgenital verwachsenen Filamente erstrecken kann. 


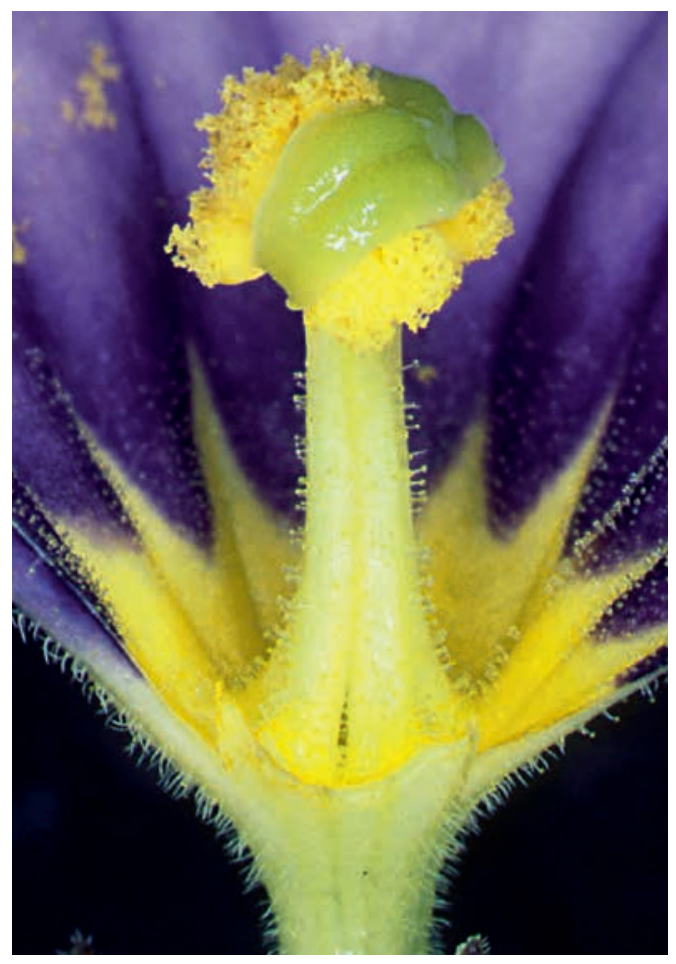

Abb. 17: Aufgeschnittene Blüte von Nierembergia scoparia (Solanaceae). Der Elaiophor besteht aus ölsezernierenden Drüsenhaaren, die sich im gelben, sternförmigen Blütenzentrum und an der Filamentsäule befinden. (Foto: G.

GerLaCH; cult. BG München-Nymphenburg)

Nierembergia gehört zu der einzigen Familie mit klassischen Ölblumen, die nicht von STEFAN Vogel entdeckt wurde. Die Entdeckung geht auf die amerikanischen Forscher Simpson und Neff (1981) zurück, die an den Blüten von $N$. gracilis Ölabscheidung beobachteten. Eingehende Studien zur Bestäubungsbiologie der Gattung führte später Cocucci 1991 durch. Er konnte feststellen, dass die meisten (aber nicht alle) Arten von Nierembergia ölblütig sind und konnte als Bestäuber Arten der Bienen-Gattungen Centris (Apidae-Apinae-Centridini) sowie Chalepogenus, Lanthanomelissa, Paratetrapedia, Tapinotaspis (Apidae-Apinae-Tapinotaspidini) ausmachen, wobei Tapinotaspis-Arten die bei weitem häufigsten Bestäuber sind. Das Titelbild der vorangehenden Ausgabe dieser Zeitschrift zierte ein Foto, das von Cocucci aufgenommen wurde und die Biene Chalepogenus parvus beim Ölsammeln an der Blüte von Nierembergia lineariifolia zeigt.

\section{Krameriaceae: Krameria}

Die etwa 18 Arten umfassende Gattung Krameria ist die einzige Gattung der Familie. Sie ist rein neotropisch verbreitet und in der Pharmazie als Lieferant der Droge Radix ratanhiae (Ratanhiawurzel) bekannt. Bei den Pflanzen handelt es sich um strauchförmige Halbparasiten, die auf den Wurzeln verschiedener Wirtspllanzen schmarotzen. Der Bau der kleinen, rosa gefärbten Blüten ist komplex, wobei Elemente des Kelches Schaufunktion übernehmen. Nach langer "Wanderschaft" (u. a. wurde die Familie mit den Polygalaceae in Verbindung gebracht) wird die Familie heute zur Ordnung der Jochblattartigen (Zygophyllales) gestellt.

Die Entdeckung, dass die Blüten Öl produzieren, geht auf Vogel (1974) zurück, der allerdings zunächst nur fixiertes Material untersuchen konnte und nur auf Grund morphologisch-anatomischer Merkmale auf die Sekretion von Öl schließen konnte. Seine Voraussagen haben sich später durch chemische Analysen des Sekretes voll bestätigt (Simpson et al. 1977, 1979; Seigler et al. 1978).

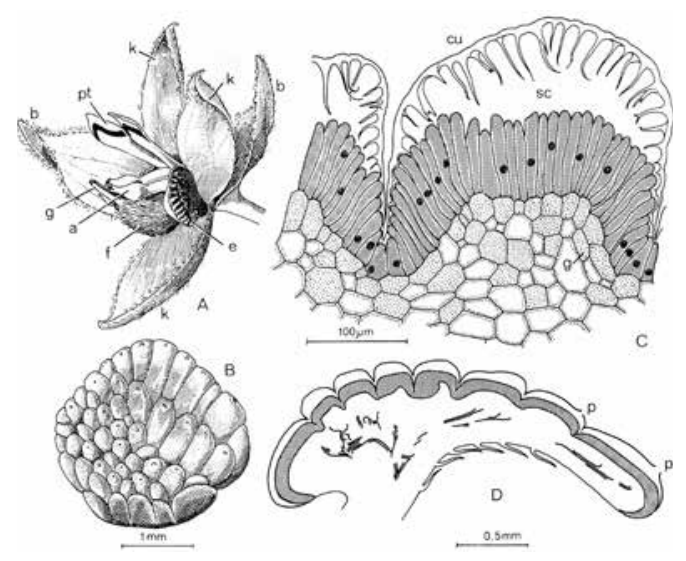

Abb. 18: Krameria triandra (Krameriaceae). A: Blüte in Seitenansicht, B: Rücken der Drüsenschuppe in Aufsicht, C: Schnitt durch eine einzelne Epithelkammer, D: Längsschnitt durch eine Drüsenschuppe; a: Anthere, b: Vorblatt, cu: Cuticula mit langen Zapfen, e: Drüsenschuppe (Elaiophor), f: Fruchtknoten, g: Griffel, g (in C): Gerbstoffzellen, k: petaloides Kelchblatt, p: Porus, pt: Petalum. (aus Vogel 1974, Abb. 28) 

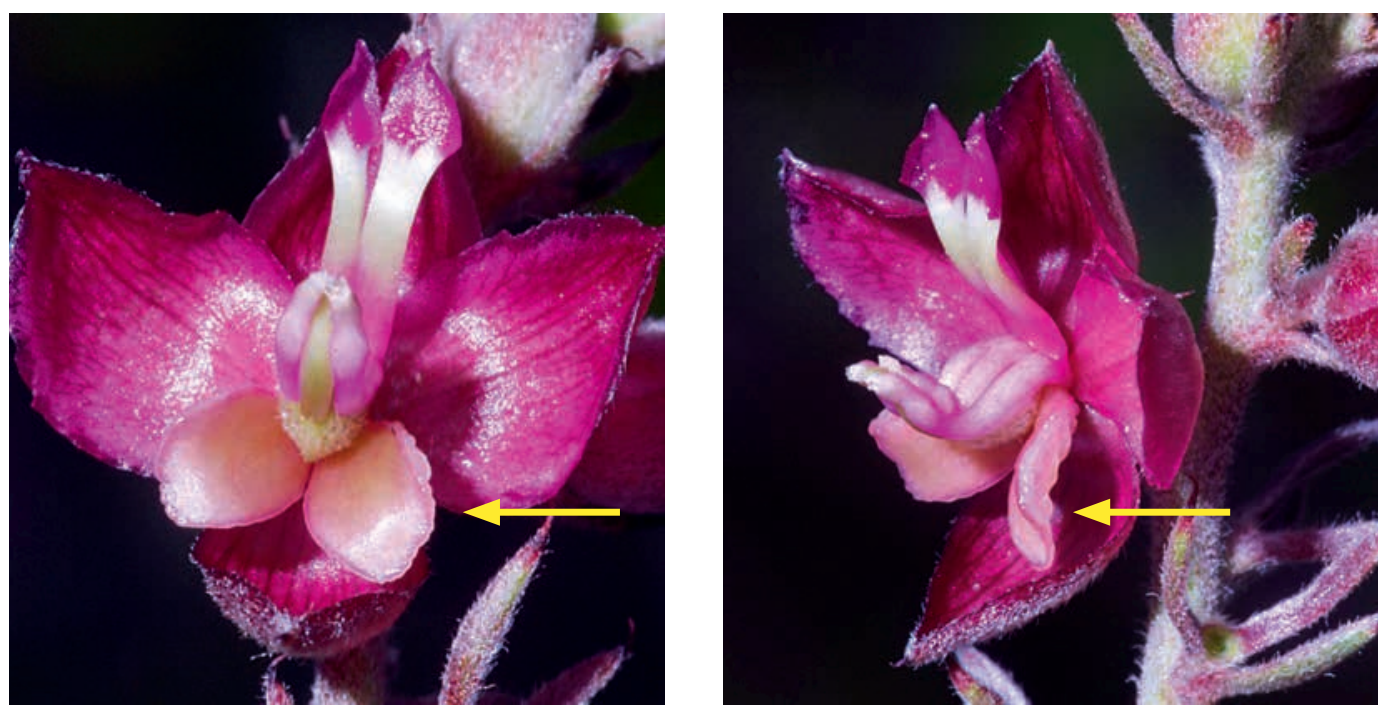

Abb. 19: Krameria tomentosa (Krameriaceae), Blüte in Vorder- (links) und in Schräg-Seitenansicht (rechts); Pfeil: Drüsenschuppe mit dem gekammerten Elaiophor auf der Rückseite; vgl. dazu auch Zeichnung und Beschriftung in Abb. 18.

(Fotos: G. Gerlach; Brasilien, Edo. Pernambuco, Catimbau)

Der Schauapparat der Blüte besteht aus vier auf der Innenseite gefärbten und kreuzförmig gestellten Kelchblättern. Die Krone umfasst je nach Art zwei oder drei adaxiale petaloide und zwei abaxial-laterale drüsige Strukturen, die so genannten Drüsenschuppen. Diese tragen auf ihrer Außen-(Rück)seite den aus zahlreichen Einzelkammern bestehenden Elaiophor. Die Anatomie der Drüsenschuppen gleicht bis in die Einzelheiten jener der Malpighiaceen-Kelchdrüsen.

Vogel (1974) konnte seinerzeit nur unvollständige Beobachtungen hinsichtlich der Bestäubung machen. Genauere Daten liegen erst aus jüngerer Zeit vor: es sind Arten der Gattung Centris, welche das Öl sammeln und als Pollenüberträger fungieren (Simpson et al. 1977; Gimenes \& Lobâo 2006, Carneiro et al. 2015, Possoвом \& MACHADO 2017, darin weitere Literatur).

\section{Malpighiaceae}

Die meisten und auch umfassendsten Beobachtungen zur Bestäubungsbiologie von Ölblumen liegen für die Malpighiaceeen vor. Obwohl das Ölblumensyndrom nicht auf die Neotropis beschränkt ist und die Verbreitung der Familie tropische Bereiche weltweit umfasst, haben sich Ölblumen nur in Lateinamerika entwickelt. Von den insgesamt etwa 1300 Arten bieten mehr als 800 fettes Öl an.

Vertreter der Malpighiaceae finden sich nur selten in tropischen Regenwäldern, sondern eher in offenen, meist trockeneren Habitaten. Es handelt sich fast immer um verholzte Pflanzen: kleine Bäume, Sträucher oder Lianen. Eine morphologische Besonderheit dieser Pflanzen sind die „malpighioiden“ (T-förmigen) Haare, die in vielen Fällen mit scharfen Spitzen für ihren wehrhaften Charakter sorgen. Die Blüten stehen meist in dichten Blütenständen. Die bei weitem vorherrschende Blütenfarbe ist ein leuchtendes Gelb, doch gibt es auch rosafarbene und weiße Blüten. Blütenduft ist nur selten wahrnehmbar. Als Fruchtformen entwickeln sich (z. T. essbare) Steinfrüchte oder Trockenfrüchte, die - ähnlich wie beim Ahorn in geflügelte Teilfrüchte zerfallen. Die Acerolakirsche (Malpighia glabra) ist eine begehrte, säuerlich schmeckende vitaminreiche Steinfrucht. Weiterhin werden verschiedene Bunchosia- und Byrsonima-Arten wegen ihrer Früchte kultiviert. Von Bedeutung ist auch die Ayahuasca-Liane, die von den Schamanen im amazonischen Tiefland wegen ihres hohen Alkaloidgehaltes als bewusstseinserweiternde Droge verwendet wird. 


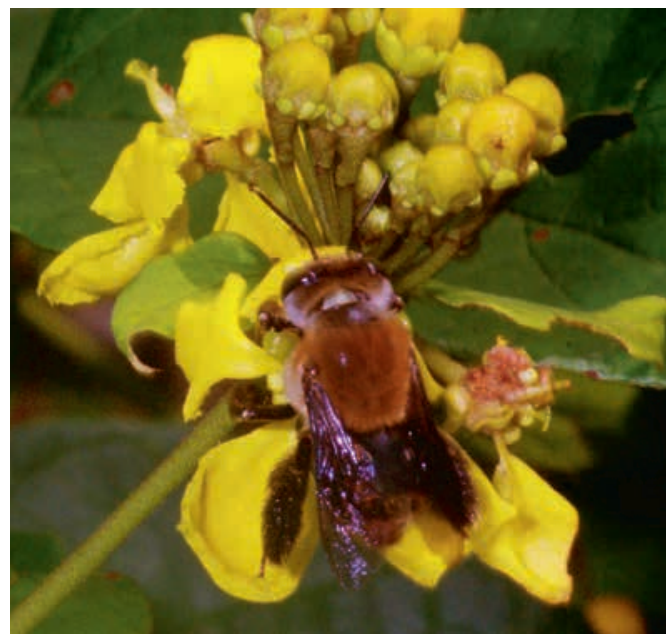

Abb. 20: Stigmaphyllon columbicum (Malpighiaceae) mit Centris aff. rubripes beim Öl-Sammelakt. Dieser dauert pro Blüte nur 2 Sekunden. Auf den Knospen sind die kelchbürtigen Öldrüsen zu erkennen. (Foto: G. Gerlach; Kolumbien, Dept. Caldas, nahe La Dorada)

Dass die lateinamerikanischen Malpighiaceen florales Öl als Attraktans für Insekten (Bienen) anbieten, nicht aber die afrikanischen und asiatischen Arten, wurde von Stefan Vogel (1974) nicht nur entdeckt, sondern er hat sich mit dieser Familie auch besonders intensiv beschäftigt. Die darauf Bezug nehmenden Publikationen und Tagungsbeiträge gehen z. T. weit über die bestäubungsbiologischen Aspekte hinaus und behandeln pflanzengeographische und evolutionshistorische Themen (z. B. Vogel 1990b). Auch einen wissenschaftlichen Film hat Vogel in Südamerika gedreht (Vogel 2002a, 2005), der heute im Internet unter der Adresse https://av.tib.eu/ media/15807 zugänglich ist.

Für das Verständnis des Ölsammelns und der Anpassungen der Blüte müssen wir etwas weiter ausholen. Den Ausgangspunkt bildet die Tatsache, dass die Malpighiaceen zahlreiche Nektarien besitzen: kaum eine Position des Pflanzenkörpers Stipeln, Blattstiel, Blattbasis, Blattrand, Brakteen, Kelchblätter, Staubblätter, Griffel - bleibt von ihnen unbesetzt.

Jene Drüsen, die auf den Kelchblättern sitzen, haben sich bei den meisten neuweltlichen Arten

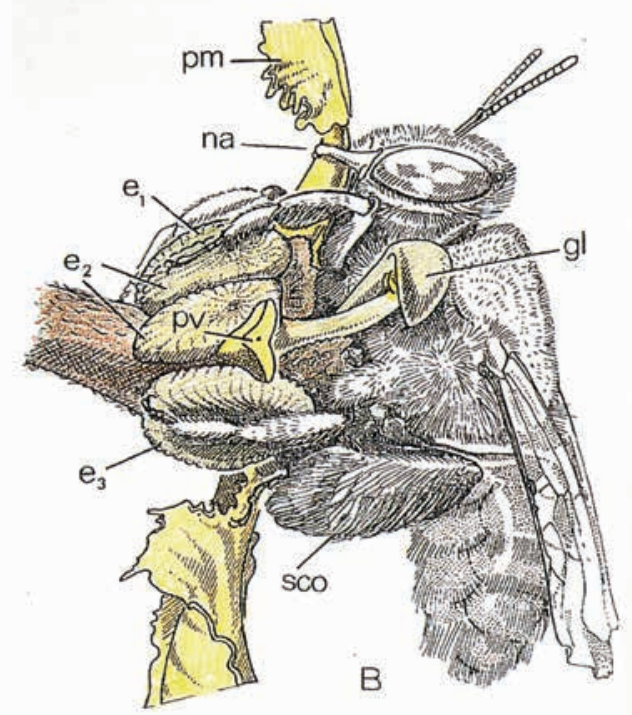

Abb. 21: Centris trigonoides (Apidae-Apinae-Centridini) beim Ölsammen auf einer Blüte von Stigmatophyllum littorale (Malpighiaceae). Die Biene hält sich mit den Mandibeln am Nagel (na) des oberen Blütenblattes (pm) fest und wischt mit den Vorder- und Mittelbeinen das Öl von den Elaiophoren (e1-4) ab. Die Hinterbeine sind nicht am Sammelakt beteiligt, doch dienen die Höschen der Hinterbeine (Scopae, sco) als Speicherorgane für das Öl und ggf. Pollen. (aus Vogel 1999, Abb. 65)

in Epithel-Elaiophoren verwandelt, die von entsprechend angepassten ölsammelnden Bienen ausgebeutet werden. Um den ungehinderten Zugriff auf diese zu gewährleisten, sind die Blütenblätter genagelt (schmaler, stielartiger Unterteil und flächiger Oberteil). So kann der Bestäuber auf der Blüte sitzend hinter die Blütenebene greifen, um an die Elaiophoren bzw. das begehrte Öl zu gelangen.

Der Sammelvorgang lässt sich kurz wie folgt beschreiben: die Biene landet auf der schwach zygomorphen Blüte und beißt sich mit ihren Mandibeln (Kiefer) am oberen, hierfür besonders ausgestalteten Blütenblatt (Vexillum) fest. Somit ist beim Ölsammeln nur eine einzige Position auf der Blüte möglich. Da sich das Tier nur mit den Mundwerkzeugen festhält, hat es alle sechs Beine frei zur Verfügung. Die Vorder- und Mittelbeine werden synchron zum Sammeln des Öls eingesetzt. Die beiden Hinterbeine werden mit Pollen und Öl beladen. Während des Ölsammelvorganges werden 
sie nach oben weggestreckt, sodass sie nicht im Weg stehen und kein Sammelgut verloren geht.

Besonders faszinierend in der Evolution dieser Elaiophoren ist deren Anzahl. Für jedes Sammelbein stehen zwei Drüsen zu Verfügung. Da die Biene ihre Position nicht auf der Blüte verändert, kann sie mit jedem Bein zwei Elaiophoren erreichen. Malpighiaceen besitzen aber als typische zweikeimblättrige Pflanzen fünfzählige Blüten, d. h. es sind je fünf Kelch- und Blütenblätter ausgebildet. Wie kommt nun die Vierzähligkeit der Elaiophoren zustande? Die beiden, dem Festhalte-Blütenblatt nächst liegenden Kelchblätter tragen jeweils nur eine Öldrüse, die anderen drei Kelchblätter aber je zwei. So bildet sich eine Summe von acht Elaiophoren, die das Kriterium der Vierzähligkeit erfüllen. Ist die Biene auf der Blüte gelandet, geht der Sammelvorgang mit einer irrwitzigen Geschwindigkeit vonstatten, sodass es schwierig ist, ihn genauer zu beobachten. Das Tier zerreißt mit den Klauen der Beine die schützende Kutikula der Öldrüsen und gelangt so an das begehrte Öl. Dieses wird mit saugfähigen Haarpolstern auf den Sammelbeinen aufgenommen und schließlich im Flug an die Hinterbeine zur sicheren Zwischenlagerung für den Heimtransport weitergegeben. Wenn nach dem Besuch von mehreren Blüten die Transportbehälter (Scopae) gefüllt sind, kehrt die Biene zu ihrer Brutzelle zurück, um dort das Sammelgut abzuladen.

Die Pollenübertragung bzw. Bestäubung erfolgt dadurch, dass die Biene beim Festklammern am Blütenblatt und beim Greifen hinter die Blütenebene auf die im Blütenzentrum positionierten Staubblätter und Narben (Antheren-Narben-Komplex) drückt, wobei sie mit Pollen beladen wird bzw. mitgebrachter Pollen auf den Narben deponiert wird.

\section{Cucurbitaceae: Momordica, Thladiantha}

Der dritte Teil der großangelegten Ölblumen-Trilogie (Vogel 1990) ist den Gattungen Momordica und Thladiantha aus der Familie der Kürbisgewächse (Cucurbitaceae) bzw. ihren Bestäubern, den Ctenoplectridae, gewidmet. Die beiden Gattungen der Ctenoplectridae (Ctenoplectra, Cteno- plectrina) werden heute nicht mehr in eine eigene Familie gestellt, sondern als Tribus Ctenoplectrini den Apidae-Apinae eingegliedert. Vogels 186 Seiten umfassende Darstellung wurde im Jahre 1990 publiziert, doch die ersten Beobachtungen reichen weit in die 1970er Jahre zurück (VogeL in Troll 1974). Nachdem Vogel bei Lysimachia einen Vertreter der Melittidae (Macropis) als Bestäuber festgestellt hatte, suchte er nach weiteren potenziell ölsammelnden Bienen-Gattungen und -Familien. Ein erster Hinweis kam von Dr. S. Peters, einem Entomologen des Senckenberg-Museums in Frankfurt am Main. Er hatte an im Museum aufbewahrten Ctenoplectra-Bienenweibchen merkwürdige Beinstrukturen beobachtet und teilte diese Beobachtung Stefan Vogel mit. Dieser stellte fest, dass es sich wahrscheinlich um Ölsammelapparate handelte, die aus spatelförmigen Anhängen an den Mittelbeinen, kammartigen Spornen an den Hintertibien und einer Scopa-artigen Bauchbürste bestanden. Der botanischen Literatur konnte Vogel erste Hinweise entnehmen, dass die Bienen wahrscheinlich an tropischen Kürbisgewächsen (die Gattungen $M o$ mordica und Thladiantha) gebunden waren. Erste Untersuchungen an Blüten von lebenden Pflanzen (männliche Pflanze von Thladiantha dubia), die im Botanischen Garten Berlin-Dahmen kultiviert wurden, ergaben keine eindeutigen Ergebnisse: die Menge des sezernierten Öls war im Vergleich zu anderen Ölblumen äußerst gering, und was vor allem irritierte: die Blüten besaßen auch ein funktionsfähiges Nektarium. Untersuchungen an einer kultivierten Momordica-Art, M. charantia, verliefen überhaupt ergebnislos: an diesen Blüten war keine Spur von Elaiophoren oder Ölsekretion zu entdecken.

VogeL wäre nicht Vogel gewesen, wenn er sich durch diese nicht gerade ermutigenden Ergebnisse hätte abhalten lassen, der Sache weiter auf den Grund zu gehen. Beharrlich sammelte er einen Mosaikstein nach dem anderen, ohne zunächst die Pflanzen und ihre Besucher am Standort gesehen zu haben. Im Jahre 1978 war es aber dann soweit: anlässlich einer Forschungsreise nach Kamerun konnte er Momordica foetida in natura studieren und Ctenoplectra-Weibchen als Ölsammler 
und Bestäuber beobachten. Da Momordica-Arten auch in Südostasien zu finden sind, sagte er voraus, dass die bisher für rein afrikanisch gehaltene Bienengattung Ctenoplectra auch dort vertreten sein müsse. 1979 unternahm er (zusammen mit dem Erstautor dieses Artikels) eine 6-wöchige Reise nach West- (Malaiische Halbinsel) und Ost-Malaysia (nördlicher Teil von Borneo). Nach wochenlanger ergebnisloser Suche nach den Pflanzen trat zwei Tage vor Abreise endlich das ersehnte Ereignis ein: in der Nähe von Sandakan (NO-Borneo) konnten blühende Pflanzen von $M$. cochinchinensis aufgefunden werden - und im gleichen Augenblick waren auch schon die Bestäuber da: die vorausgesagten Ctenoplectra-Bienen. 1981 trat Vogel mit einer kurzen Publikation an die Öffentlichkeit, in der er die neuartige Methode

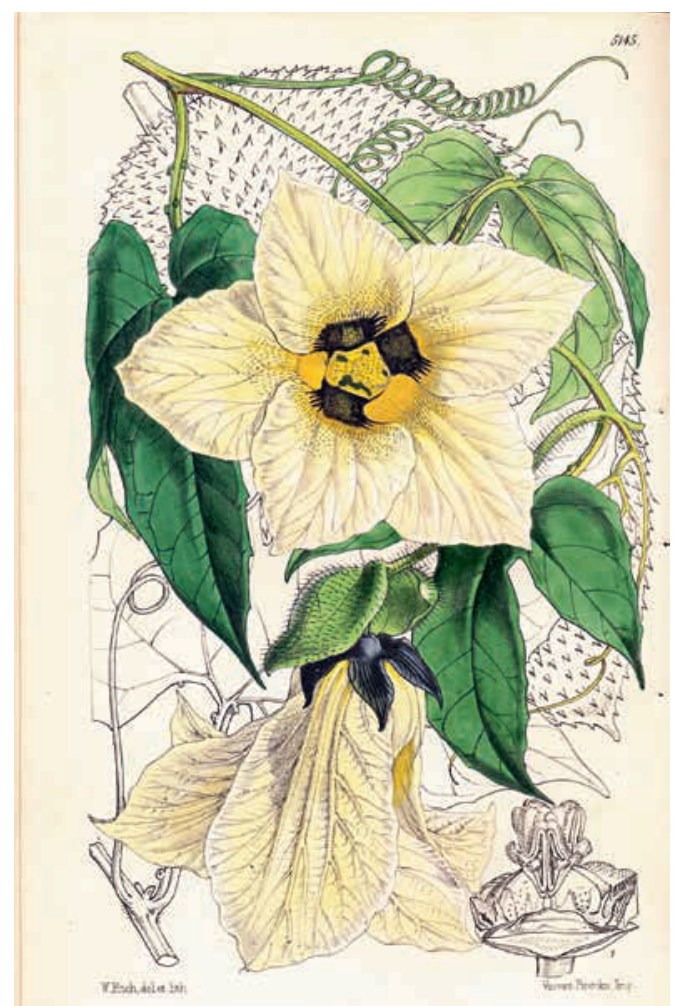

Abb. 22: Momordica cochinchinensis (Cucurbitaceae; als M. mixta bezeichnet). Blühende männliche Pflanze; die Öldrüsenfluren (Trichom-Elaiophoren) befinden sich auf dem unteren Teil der Kronblätter (die Drüsen sind als feine, schwarze Strichlein zu erkennen); zentraler Teil einer weiblichen Blüte in der Zeichnung links unten. (VAN Houtte, Flore des serres et des jardins de l'Europe, vol. 14: t. 0, 1845) des Ölsammelns von Ctenoplectra vorstellte: Die Bienen-Weibchen laufen über die ölsezernierenden Drüsenfelder und bewegen dabei den Hinterleib hin und her (Ölschwänzeln). Das Öl wird mit der haarigen Unterseite des Abdomens (Bauchbürste) aufgesaugt und mit Hilfe der Mittelbeine in die Höschen (Scopae) der Hinterbeine transferiert.

Nach einer dritten Reise (1986), die Vogel wieder nach Westafrika (Togo) führte und bei der er weitere Gelände-Beobachtungen zur $\mathrm{Mo}$ mordica-Bestäubung machen konnte, sowie nach ausführlichen Laborstudien (Studium der Morphologie und Anatomie der gesammelten $\mathrm{Mo}$ mordica- und Thladiantha-Blüten, Ultrastruktur der ölabscheidenden Drüsen, chemische Analysen des Blütenöls etc.) folgte die Niederschrift und $\mathrm{Pu}-$

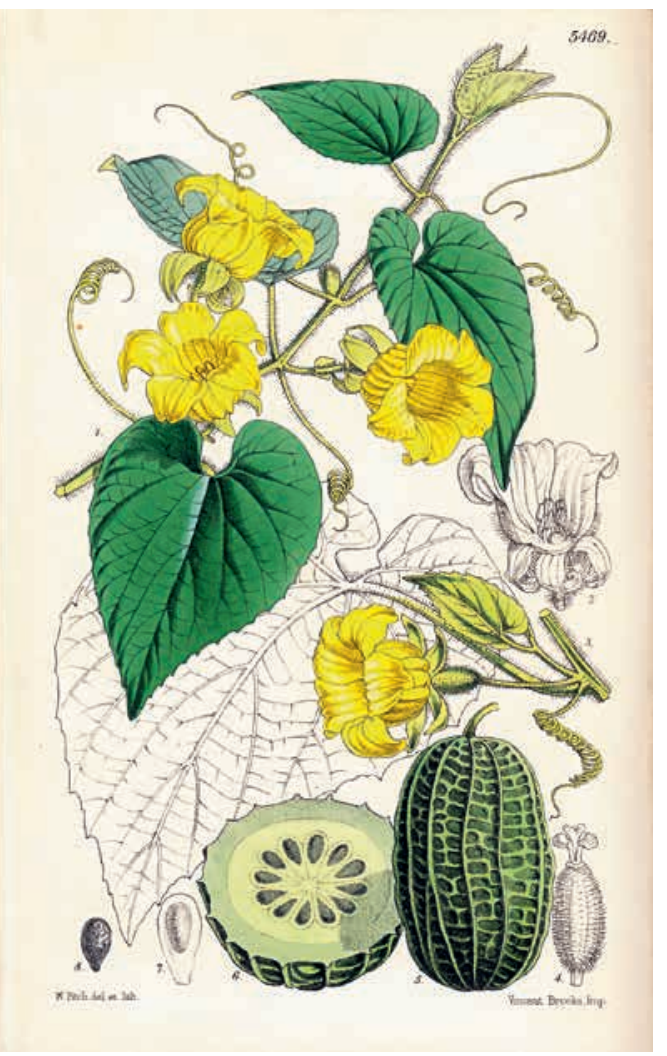

Abb. 23: Thladiantha dubia (Cucurbitaceae), blühende männliche Pflanze (oben) und aufgeschnittene männliche Blüte (Zeichnung Mitte rechts), sowie weibliche Blüte (oberhalb der Frucht); die glockenförmigen Blüten tragen am Glockengrund die Trichom-Elaiophoren. (aus Curtis's Bot. Mag., vol. 90, t. 5469, 1864) 


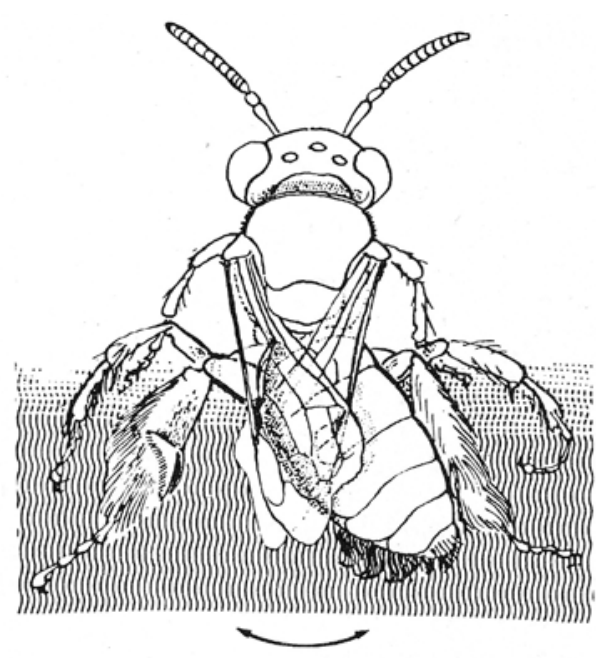

Abb. 24: Weibliche Biene von Ctenopletctra beim Aufsammeln des Blütenöls. Der Hinterleib mit der unterseitigen Bauchbürste wird in rascher Folge hin- und her bewegt. (aus Vogel 1981, Fig. 2)

blikation der Ergebnisse in dem schon erwähnten dritten Teil der Ölblumentrilogie.

Die Ergebnisse von Vogels Studien lassen sich, ergänzt durch neuere Befunde, folgendermaßen zusammenfassen:

(1) Bei den Kürbisgewächsen bieten die Gattungen Momordica (ca. 60 Arten, Schwerpunkt Westafrika, 47 Arten, östlich über Süd- und Südostasien bis Neuguinea und Australien, 12 Arten, Schaefer \& Renner 2010) und Thladiantha (> 20 Arten, subtropisches und warm-temperates China) fettes Öl als florales Attraktans an (auf einige wenige Ausnahmen werden wir noch zu sprechen kommen). Dazu kommt die Gattung Indofevillea, deren zwei Arten florales Öl als Attraktans darbieten (SCḦ̈FER et al. 2012). Nach jüngsten und noch unpublizierten Beobachtungen von $\mathrm{S}_{\mathrm{CH}} \ddot{\mathrm{A}}$ FER (siehe RenNer \& SCHÄFER 2010) gehören noch drei weitere Cucurbitaceen-Gattungen in die Gruppe der Ölblumen: Siraitia, Telfairia und Baijiania incl. Sinobaijiania, alle Asien, mit jeweils 3-5 Arten).

(2) Das florale Öl wird ausschließlich von den (insgesamt >30) Arten der Bienengattung Ctenoplectra gesammelt. Soweit bekannt, sind alle Ctenoplec-

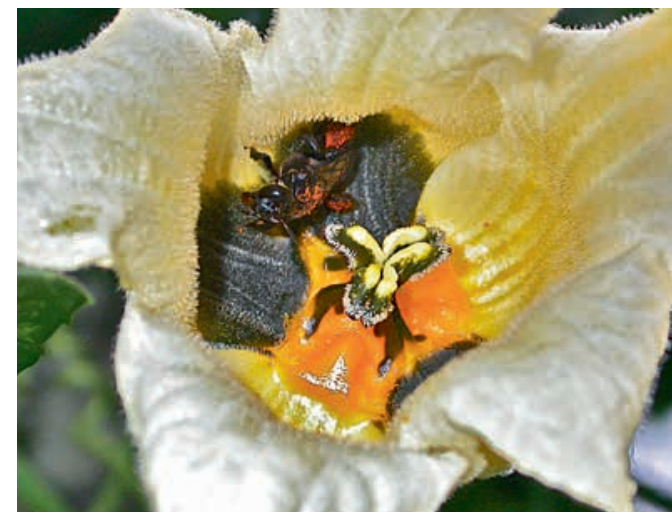

Abb. 25: Männliche Blüte von Momordica cochinchinensis (Cucurbitaceae) mit der Biene Ctenoplectra australica (Apidae-Apinae-Ctenoplectrini). An der Umschlagstelle des linken Kronblattes ist deutlich die Öldrüsenflur (Trichom-Elaiophor) zu erkennen. Die Biene trägt an mehreren Stellen orangefarbenen Pollen auf dem Körper. (Foto: H. Schäfer; Australien, Queensland, Iron Range National Park)

tra-Arten auf das Sammeln von Öl spezialisiert. Bei der zweiten Gattung der Ctenoplectrini, Ctenoplectrina (3 Arten in Afrika), ist dieses Verhalten wahrscheinlich sekundär verloren gegangen (Renner \& Schaefer 2010).

(3) Die Drüsenfelder (Elaiophoren) befinden sich auf den Basen der Kronblätter (Petalen). Die Elaiophoren sind bei Thladiantha von gleicher Farbe wie die gesamte Blüte (im UV-Licht jedoch durch die starke UV-Absorption auffällig). In beiden Fällen bestehen sie aus Fluren von vielzelligen, kurzstielig-keulenförmigen Drüsen.

(4) Da die Blüten der Cucurbitaceen eingeschlechtig (mit monözischer oder diözischer Verteilung) sind, müssen wir die männlichen und weiblichen Blüten gesondert betrachten. Bei den männlichen Blüten von Momordica lassen sich grob zwei Typen unterscheiden: (a) Blüten \pm radiär, flach und gelb; auf jedem Petalum befindet sich ein Elaiophor. Zusätzlich befinden sich auf den beiden äußeren Petalen Nektarien, deren Zugang von einer Ligula-artigen Basalschuppe geschützt wird. (b) Blüten ausgesprochen asymmetrisch, tiefschlundig und creme-weißlich gefärbt. Die beiden äußeren, größeren Petalen $(1,2)$ tragen an der Basis ein Nektarium mit Basalschuppe. Die beiden innersten 
Petalen $(4,5)$ besitzen kein Nektarium, sondern tragen lediglich einen schwärzlichen Fleck. Petalum 3 nimmt eine intermediäre Stellung ein: es hat hälftig einen schwarzen Fleck und ein Nektarium, dieses jedoch ohne Nektarzugang. Die schwarzen Flecken in den Blüten dienen als Ruhestellen für die ebenfalls schwarzen Bienen, die hier von Fressfeinden nicht so leicht zu entdecken sind (HANNO SCHÄFER, pers. Mitt.). Bei Thladiantha tragen alle fünf Petalen einen Elaiophor, jedoch hat in der Regel nur Petalum 2 ein Nektarium bzw. eine Basalschuppe (seltener zusätzlich auch das erste Petalum).

(5) Die weiblichen Blüten tragen nie ein funktionsfähiges Nektarium, doch sind Rudimente von solchen bzw. rudimentäre Basalschuppen vorhanden. Die Blüten bieten ausschließlich Öl als Sammelgut an. Die Elaiophoren bestehen aus schütteren (Thladiantha) bis dichten und gut abgegrenzten (Momordica) Drüsenfluren an der Basis der Petalen. Sie sind vergleichsweise wenig ergiebig, können aber bei Momordica auch größere Öltropfen bilden. Die Elaiophoren sind meist in Fünfzahl vorhanden.

(6) Einige wenige Momordica-Arten, wie die in den gesamten Tropen als Gemüse- und Heilpflanzen kultivierten Bittermelonen (M. charantia, M. balsamina), besitzen keine Elaiophoren bzw. florales Öl, sondern sind reine Nektarblumen. Es handelt sich jedoch hier nicht um ursprüngliche Arten, die sozusagen die Ölproduktion noch nicht erfunden haben, sondern um abgeleitete Taxa, bei denen die Ölsekretion im Laufe der Evolution sekundär verloren gegangen ist. Diese schon von VoGEL aufgestellte These hat jüngst durch molekular-systematische Analysen ihre Bestätigung gefunden (Renner \& Schäfer 2010). Die Fähigkeit zur Bildung floralen Öls wurde bei den Cucurbitaceen nur einmal erworben, ist aber mehrfach parallel wieder verloren gegangen.

(7) Der Nektar der männlichen Blüten wird von beiden Geschlechtern der Ctenoplectra-Bienen aufgenommen. Er dient der Eigenversorgung und Energiegewinnung. Bei der Nektaraufnahme wird Pollen auf dem Thorax der Bienen aufgebracht, wo er außer Reichweite der Putzbewegungen bleibt. Das Blütenöl wird hingegen nur von den Bienenweibchen gesammelt. Bringt ein Weibchen Pollen einer männlichen Blüte mit, wird dieser an den Narben abgestreift. Damit ist die Pollenübertragung vollzogen. Durch die Bereitstellung sowohl von Pollen, Nektar und Öl in den männlichen Blüten und Öl in den weiblichen Blüten werden die Ctenoplectra-Bienen an die Blüten von Momordica und Thladiantha gebunden. Die Lebenszyklen der Pflanzen und der Bienen sind somit perfekt aufeinander abgestimmt.

\section{Primulaceae: Lysimachia- Ölblumen vor unserer Haustür}

Nach den bisherigen Ausführungen mag der Eindruck entstanden sein, dass Ölblumen und ihre Bestäubung durch hochspezialisierte Bienen ein rein tropisches Phänomen darstellen. Dem ist aber keineswegs so. Auch in unserer heimischen Flora gibt es Ölblumen, sogar mehrere Arten. Alle gehören der Gattung Lysimachia (Gilbweiderich) aus der Familie der Primelgewächse an.

Insgesamt umfasst die Gattung Lysimachia ca. 180 Arten (ANDERberg et al. 2007) und davon bilden etwa $40 \%$ Ölblumen. Sie sind die einzigen Vertreter der Ölblumen in Europa, Nordamerika und weiten Teilen Asiens. Bestäubt werden diese Primelgewächse ausschließlich von Ölbienen der Gattung Macropis (Melittidae-Melittinae). Eine erste Erwähnung, dass Pflanzen dieser Gattung Ölblumen darstellen, findet sich in Troll (1972: 101), der über Forschungsergebnisse von VogeL folgendermaßen berichtet: „Inzwischen konnte Prof. Vogel den Kreis bekannter Ölblumen durch die unerwartete Entdeckung ausweiten, daß dieses Pollinationsprinzip auch im holarktischen Bereich vorkommt, und zwar in mindestens drei Sektionen der Gattung Lysimachia, inklusive L. vulgaris, L. punctata und L. nummularia. Diese galten bisher als Pollenblumen. Die Existenz eines Drüsenhaarbesatzes war zwar schon früher aufgefallen, das Sekret jedoch für eine terpenartige Substanz gehalten und eine ökologische Bedeutung desselben in Abrede gestellt worden. 


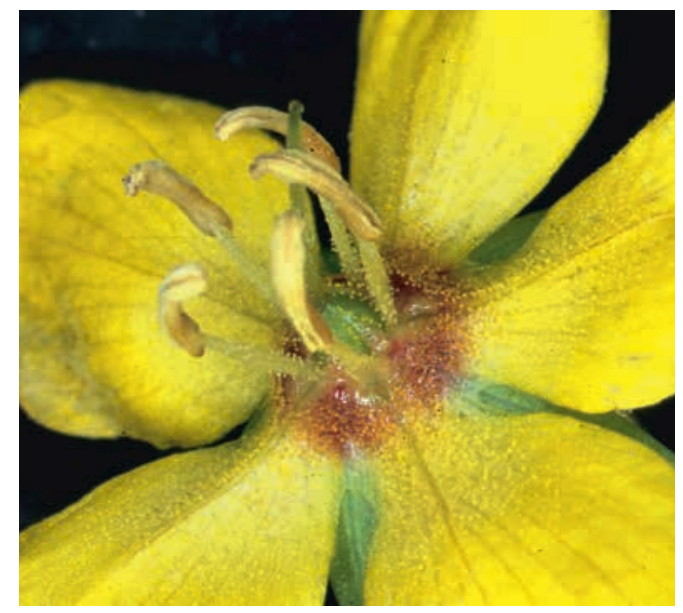

Abb. 26: Lysimachia ciliata (Primulaceae). Die ölsezernierenden Drüsen (Elaiophor) sind im Zentrum der Blüten, auf den Basen der Kronblätter und auf den Filamenten gut zu erkennen.. (Foto: G. Gerlach, BG München-Nymphenburg)

Lysimachia ist seit längerem als nahezu alleiniger Wirt der Bienengattung Macropis (Melittidae) bekannt. Diese hatte Verwunderung durch die Eigenart erregt, ihre Pollenladungen zu benetzen, was im weiteren Verwandtschaftskreis sonst nicht vorkommt". Nach der Erkenntnis, dass Lysimachia-Arten Ölblumen und Macropis-Bienen Ölbienen darstellen (erste Mitteilung: Vogel 1976), hat sich Vogel intensiv mit der Blütenbiologie (Blütenbau, chemische Zusammensetzung des Öls) mitteleuropäischer, asiatischer und nordamerikanischer Vertreter von Lysimachia, der Biologie der Schenkelbienen (Morphologie, Nestbau, Nektarpflanzen) sowie der Interaktion der beiden Partner (Pollen- und Ölsammelverhalten, Bestäubung, Bestäuberanlockung) befasst. Sein diesbezügliches Hauptwerk stellt das von der Akademie der Wissenschaften und der Literatur in Mainz publizierte Buch „Ölblumen und ölsammelnde Bienen, zweite Folge: Lysimachia und Macropis" dar (Vogel 1986). Zu erwähnen ist auch sein IWF-Film „Gilbweiderich und Schenkelbiene“ (Vogel 2002c, 2005), der im Internet unter der Adresse https://av.tib.eu/ media/15806 abgerufen werden kann.

In der Natur lassen sich der Blütenbesuch und die Bestäubung durch Schenkel-Bie-

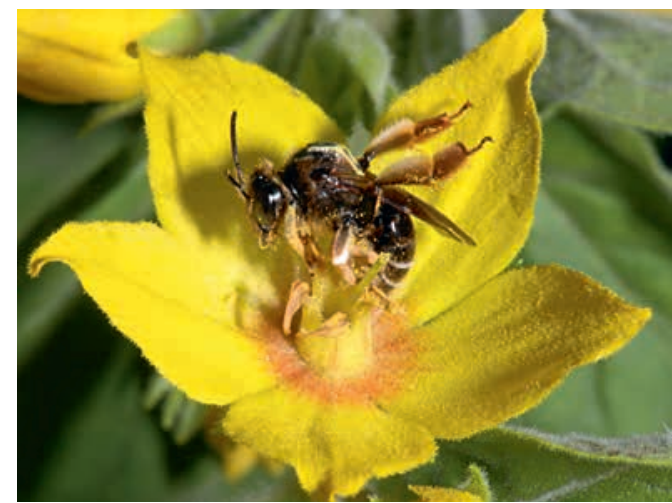

Abb. 27. Ein frisch geschlüpftes und am Thorax markiertes Weibchen der Wald-Schenkelbiene (Macropis fulvipes) (Melittidae) auf einer Blüte von Lysimachia punctata. Die Hinterbeine werden während des Blütenbesuchs hochgestreckt. Deutlich sichtbar ist die dichte Behaarung auf den Schienen (Tibien) und Fersengliedern (Metatarsus) zu sehen, da die Biene noch keinen Pollen und Öl gesammelt hat. (Foto: S. DötTerL)

nen ( $M$. europaea, Auen-Schenkelbiene, und M. fulvipes, Wald-Schenkelbiene) am besten bei L. vulgaris (Gewöhnlicher Gilbweiderich) und L. punctata (Punktierter Gilbweiderich) beobachten. Erstere Art ist eine in Mitteleuropa häufige Pflanze, die in Feuchtbiotopen (Feuchte bis nasse, meist basenreiche Wiesen- und Waldstandorte, Ufer- Gebüsche, Erlenbruchwälder etc.) vorkommt, während letztere seltener anzutreffen ist (Wald- und Gebüschränder, Hochstaudenfluren, Feuchtwiesen), aber häufig als Zierpflanze in Gärten kultiviert wird. Hingegen wird die kriechende L. nummularia (Pfennigkraut) nur selten von Bienen besucht, und selbst wenn, bildet sie kaum Früchte bzw. reife Samen aus. Diese Pflanzenart breitet sich vorwiegend vegetativ, durch Bildung von langen Ausläufern, aus.

Die Arbeiten von Vogel waren Basis für weiterführende Arbeiten zur Bionomie der Schenkelbienen (CANe et al. 1983, Celary 2004, Schäffler \& Dötterl 2011), der Zusammensetzung des Blütenöls (SEIPold 2004), sowie der Kommunikation zwischen Lysimachia und Macropis (Dötterl \& Schäffler 2007, Dötterl et al. 2011, Schäffler et al. 2012, SCHäFfLER et al. 2015). 
Sehr detailliert wurde das Verhalten von Macropis fulvipes nach dem Schlupf und beim Versorgen der Brutzellen von Schäffler \& Dötterl (2011) analysiert. Sie führten die Beobachtungen in einem Flugkäfig durch, in den Bienen aus einem Wurzelteller schlüpften. Nachdem beobachtet wurde, dass $M$. fulvipes in diesem Wurzelteller im Ökologisch-Botanischen Garten der Universität Bayreuth nistet, wurde dieser im darauffolgenden Frühjahr vor dem Schlupf der Bienen in ein Gewächshaus transportiert und der Flugkäfig rundherum gebaut. Die Untersuchungen zeigten, dass beide Geschlechter der Bienen nach dem Schlüpfen Blüten von Lysimachia besuchen, um Pollen zu fressen. In und an den Blüten übernachteten sie auch, zum Teil in großen Gruppen, die Weibchen allerdings nur solange sie kein eigenes Nest hatten. Ein solches legen sie nach dem Pollenfressen an. Ist eine Brutzelle gegraben, kleiden sie diese mit Blütenöl aus, das sie von Lysimachia-Blüten sammeln. Bei diesen Blütenbesuchen gilt das Augenmerk ausschließlich dem Öl. Erst wenn eine Brutzelle nach etwa vier Sammelflügen ausgekleidet ist und das Öl zur Bildung einer festen Schutzhülle durch Speichelsekrete verfestigt wurde, sammeln die Weibchen als Futter für die Larven in 6-8 Sammelflügen neben dem Öl auch aktiv den Pollen. Bei optimalen Bedingungen, wie reichlich blühenden Gilbweiderich in der Nähe der Nester und sonnig-warmes Wetter, benötigen die Bienen vier Stunden für die Auskleidung und Verproviantierung der Brutzellen. Sie können somit maximal 2 Brutzellen pro Tag fertigstellen und besuchen dabei in etwa 900 Blüten des Gilbweiderichs.

In unregelmäßigen Abständen trinken die Weibchen während des Nestbaus zur Eigenenergieversorgung an Nektar führenden Pflanzen wie Storchschnabelarten.

Neben dem Nestbau und Sammelverhalten wurde in dem Flugkäfig auch untersucht, wie die Kommunikation zwischen Lysimachia und den Bienen aussieht und welche Blütensignale des Gilbweiderichs für die Wirtspflanzenfindung von den Bienen genutzt werden. Sind Weibchen zum ersten Mal in ihrem Leben auf der Suche nach
Lysimachia, nutzen sie primär Duftsignale der blühenden Pflanzen und erst, wenn sie die Pflanzen riechen, auch visuelle Signale (z. B. Blütenfarbe), um diese zu finden (Dötterl et al. 2011). Später sind Duft- und visuelle Signale von ähnlich großer Bedeutung für die Bienen. Die kürzliche Identifizierung von Diacetin, einem Blütenduft von Lysimachia, als Schlüsselsubstanz für die Anlockung von Macropis, stellt generell den ersten identifizierten Lockstoff einer Ölblume-Ölbiene-Interaktion dar (SCHÄFfLER et al. 2015). Diese Substanz kommt auch in afrikanischen und neotropischen Ölblumen vor, ist chemisch dem fetten Öl sehr ähnlich, und könnte ein wichtiges Signal nicht nur für Macropis, sondern auch für weitere Ölbienen darstellen.

\section{Verwendete und weiterführende Literatur}

Aguiar, A. J. C. \& Melo, G. A. R. 2009: Notes on oil sources for the bee genus Caenonomada (Hymenoptera, Apidae, Tapinotaspidini). - Rev. Brasil. Entomol. 53: 154-156.

Anderberg, A. A., Manns, U. \& Källersjö, M. 2007: Phylogeny and floral evolution of the Lysimachieae (Ericales, Myrsinaceae): evidence from $n d h F$ sequence data. - Willdenowia 37: 407-421.

Andersson, S. 2006: On the phylogeny of the genus Calceolaria (Calceolariaceae) as inferred from ITS and plastid matK sequences. - Taxon 55: 125-137

Cane, J. H., Eickwort, G. C., Wesley, F. R. \& Spielholz, J. 1983: Foraging, grooming, and mating behaviors of Macropis nuda (Hymenoptera: Melittidae) and use of Lysimachia ciliata (Primulaceae) oils in larval provisions and cell lining. - Amer. Midl. Nat. 110: 257-264.

Carneiro, L. T., Aguiar, A. J.C., Martins, C. F., MachaDo, I. C. \& Alves-dos-Santos, I. 2015: Krameria tomentosa oil flowers and their pollinators: bees specialized on trichome elaiophores exploit its epithelial oil glands. - Flora 215: 1-8.

Celary, W. 2004: A comparative study on the biology of $M a$ cropis fulvipes (Fabricius, 1804) and Macropis europaea Warncke 1973 (Hymenoptera: Apoidea: Melittidae). - Folia Biol. Krak. 52(1-2): 81-85.

Cocucci, A. A. 1991: Pollination biology of Nierembergia (Solanaceae). - Plant Syst. Evol. 174: 17-35.

Dötterl, S., Milchreit, K. \& Schäffler, I. 2011: Behavioural plasticity and sex differences in host finding of a specialized bee species. - J. Comp. Physiol. A 197: 1119-1126.

DötTerl, S., SChäFfler, I. 2007: Flower scent of floral-oil producing Lysimachia punctata as cue for the oil-bee Macropis fulvipes. - J. Chem. Ecol. 33: 441-445. 
Gimenes, M. \& LobÃo, C. S. 2006: A polinização de Krameria bahiana B.B.Simpson (Krameriaceae) por abelhas (Apidae) na Restinga, BA. - Neotrop. Entomol. 35: 440-445.

Machado, I. C., Vogel, S. \& Lopes, A. V. 2002: Pollination of Angelonia cornigera Hook. (Scrophulariaceae) by long-legged, oil-collecting bees in NE Brazil. - Plant Biol. 4: 352-359.

Mayr, E. M. \& Weber, A. 2006: Calceolariaceae: floral development and systematic implications. - Amer. J. Bot. 93: 327-343.

Olmstead, R. G., dePamphilis, C. W., Wolfe, A. D., Young, N. D., Elisons, W. J. \& Reeves, P. A. 2001: Disintegration of the Scrophulariaceae. - Amer. J. Bot. 88: 348-361.

Pauw, A., Kahnt, B., Kuhlmann, M., Michez, D., Montgomery, G. A., Murray, E. \& Danforth, B. N. 2017: Long-legged bees make adaptive leaps: linking adaptation to coevolution in a plant-pollinator network. - Proc. Roy. Soc. B 284(1707).

Possobom, C. C. F. \& Machado, S. R. 2017: Elaiophores: their taxonomic distribution, morphology and functions. Acta Bot. Brasil. 31: 503-524.

Renner, S. S. \& Schaefer, H. 2010. The evolution and loss of oil-offering flowers - new insights from dated phylogenies for angiosperms and bees. - Phil. Trans. Roy. Soc. London, B, 365: 423-435.

Schaefer, H., Bartholomew, B. \& Boufford, D. E. 2012: Indofevillea jiroi (Cucurbitaceae), a new floral oil producing species from northeastern Myanmar. - Harvard Papers Bot. 17: 323-332.

Schaefer, H. \& Renner, S. S. 2008: A phylogeny of the oil bee tribe Ctenoplectrini (Hymenoptera: Anthophila) based on mitochondrial and nuclear data: evidence for Early Eocene divergence and repeated out-of-Africa dispersal. - Mol. Phylogenet. Evol. 47: 799-811.

Schaefer, H. \& Renner, S. S. 2010: A three-genome phylogeny of Momordica (Cucurbitaceae) suggests seven returns from dioecy to monoecy and recent long-distance dispersal to Asia. - Mol. Phylogen. Evol. 54: 553-560.

Schäffler, I., Balao, F. \& Dötterl, S. 2012: Floral and vegetative cues in oil and non-oil secreting Lysimachia species. Ann. Bot. 110: 125-138.

SChäffler, I. \& Dötterl, S. 2011: A day in the life of an oil bee: Phenology, nesting and foraging behaviour. - Apidologie 42: 409-424.

Schäffler, I., Steiner, K. E., Haid, M., van Berkel, S. S., Gerlach, G., Johnson, S. D., Wessjohann, L. \& Dötterl, S. 2015: Diacetin, a reliable cue and private communication channel in a specialized pollination system. Scientific Reports 5: 1-11; Art. no. 12779.

Seigler, D., Simpson, B. B., Martin, C. \& Neff, J. L. 1978: Free 3-acetoxy fatty acids in floral glands of Krameria species. - Phytochemistry 17: 995-996.

Seipold, L. 2004: Blütenöle - Chemische Analyse, Biosynthese und Betrachtungen zur Entstehung von Ölblumen. - Diss. Univ. Halle-Wittenberg.
Sérsic, A. \& Cocucci, A. A. 1996: A remarkable case of ornithophily in Calceolaria: food bodies as rewards for a nonnectarivorous bird. - Bot. Acta 109: 172-176.

Sérsic, A. N. 2004: Pollination in the genus Calceolaria. Stapfia 82: 1-121.

Sérsic, A. \& Cocucci, A. A. 1999: An unusual kind of nectary in the oil flowers of Monttea: its structure and function. - Flora 194: 393-404.

Simpson, B. B. \& NefF, J. L. 1981: Floral rewards: alternatives to pollen and nectar. - Ann. Missouri Bot. Gard. 68: 301-322.

Simpson, B. B., Neff, J. L. \& Dieringer, G. 1990. The production of floral oil by Monttea (Scrophulariaceae) and the function of tarsal pads in Centris bees. - Plant Syst. Evol. 173: 209-222.

Simpson, B. B., Neff, J. L. \& Seigler, D. 1977: Krameria, free fatty acids and oil-collecting bees. - Nature 267: 150-151.

Steiner, K. E. 1989: A second species of the amphi-Atlantic genus Alonsoa (Scrophulariaceae) in South Africa. - Ann. Missouri Bot. Gard. 76: 1152-1159.

Steiner, K. E. 1990: The Diascia (Scrophulariaceae) window: an orientation cue for oil- collecting bees. - Bot. J. Linn. Soc. 102: $175-195$.

Steiner, K. E. 1993: Has Ixianthes (Scrophulariaceae) lost its special bee? - Plant Syst. Evol. 185: 7-16.

Steiner, K. E., Whitehead, V. B. 1988: The association between oil-producing flowers and oil-collecting bees in the Drakensberg of Southern Africa. - Monogr. Syst. Bot. Missouri Bot. Gard. 25: 259-277.

Steiner, K. E. \& Whitehead, V. B. 1990: Pollinator adaptation to oil-secreting flowers - Rediviva and Diascia. - Evolution 44: 1701-1707.

Steiner, K. E. \& Whitehead, V. B. 1991: Oil flowers and oil bees: further evidence for pollinator adaptation. - Evolution 45: 1493-1501.

Steiner K. E. \& Whitehead V. B. 1996: The consequences of specialization for pollination in a rare South African shrub, Ixianthes retzioides (Scrophulariaceae). - Plant Syst. Evol. 201: 131-138.

Steiner, K. E. \& Whitehead, V. B. 2002: Oil secretion and the pollination of Colpias mollis (Scrophulariaceae). - Plant Syst. Evol. 235: 53-66.

TAdey, M. 2011. Reproductive biology of Monttea aphylla (Scrophulariaceae). - Austral. J. Bot. 59:713-718.

Troll, W., 1972: Kommissionsbericht für Botanische Forschung. - Jahrb. Akad. Wiss. Lit. Mainz 1972: 98-106.

Troll, W., 1974:Kommissionsbericht für Botanische Forschung. - Jahrb. Akad. Wiss. Lit. Mainz 1974: 128-142.

Vogel, S. 1974: Ölblumen und ölsammelnde Bienen. - Abh. Akad. Wiss. Lit. Mainz, Math.-Naturwiss. Kl., Reihe Tropische und subtropische Pflanzenwelt 7: 285-547. Wiesbaden. 
Vogel, S. 1976: Lysimachia: Ölblumen der Holarktis. - Naturwiss. 63: 44.

Vogel, S. 1986: Ölblumen und ölsammelnde Bienen. Zweite Folge: Lysimachia und Macropis. - Abh. Akad. Wiss. Lit. Mainz, Math.-Naturwiss. Kl., Reihe Tropische und subtropische Pflanzenwelt 54: 149-312. Wiesbaden.

Vogel, S. 1990a: Ölblumen und ölsammelnde Bienen. Dritte Folge. Momordica, Thladiantha und die Ctenoplectridae. Abh. Akad. Wiss. Lit. Mainz, Math.-Naturwiss. Kl., Reihe Tropische und subtropische Pflanzenwelt 73: 1-186. Wiesbaden.

Vogel, S. 1990b: History of the Malpighiaceae in the light of pollination ecology. - Mem. New York Bot. Gard. 55: $130-142$.

Vogel, S. 1999: Von Ölblumen und Parfümblumen. - In: Zizka, G. \& Schneckenburger, S. (Hrsg.): Blütenökologie: faszinierendes Miteinander von Pflanzen und Tieren. - Kleine Senckenberg-Reihe Nr. 33: 74-87. Zugleich Palmengarten Sonderheft Nr. 31. Frankfurt.

Vogel, S. 2002a: Ölblumen und ölsammelnde Bienen: Malpighiaceae und ihre Bestäuber. - Video-Film Nr. W 7047: IWF Göttingen: https://av.tib.eu/media/15807

Vogel, S. 2002b: Ölblumen und ölsammelnde Bienen: Die Bestäubung von Angelonia hirta (Scrophulariaceae). - Video-Film Nr. W 7048, IWF, Göttingen: https://av.tib.eu/ media/ 15808

VogeL, S. 2002c: Ölblumen und ölsammelnde Bienen: Gilbweiderich und Schenkelbiene. — Video-Film Nr. W 7049 , IWF, Göttingen: https://av.tib.eu/media/15806
Vogel, S. 2005: Malpighiaceae und ihre Bestäuber, W 7047. Die Bestäubung von Angelonia (Scrophulariaceae), W 7048. Gilbweiderich und Schenkelbiene, W 7049. DVD Version (German, English). IWF Wissen und Medien GmbH, Leibniz Gemeinschaft, Göttingen.

Vogel, S. \& Cocucci, A. A. 1995: Pollination of Basistemon (Scrophulariaceae) by oil-collecting bees in Argentina. - Flora 190: 353-363.

Vogel, S. \& Machado, I. S. 1991: Pollination of four sympatric species of Angelonia (Scrophulariaceae) by oil-collecting bees in NE-Brazil. - Plant Syst. Evol. 178: 153-178.

Whitehead, V. B. \& Steiner, K. E. 1992: Two new species of oil-collecting bees of the genus Rediviva from the summer rainfall region of South Africa (Hymenoptera, Apoidea, Melittidae). - Ann. South African Mus. 102: 4143-4164.

Whitehead, V. B. \& Steiner, K. E. 2001: Oil-collecting bees of the winter rainfall area of South Africa (Melittidae, Rediviva). - Ann. South African Mus. 108: 1-277.

\section{Anschriften der Autoren}

Prof. Dr. Stefan Dötterl, AG Ökologie, Biodiversität und Evolution der Pflanzen, Universität Salzburg, Hellbrunner Straße 34, A-5020 Salzburg, E-Mail: Stefan.Doetterl@ sbg.ac.at

Dr. Günter Gerlach, Botanischer Garten MünchenNymphenburg, Staatliche Naturwissenschaftliche Sammlungen Bayerns (SNSB), Menzinger Str. 61, D-80638 München, E-Mail: gerlach@extern.lrz-muenchen.de Prof. i. R. Dr. Anton Weber, Department für Botanik und Biodiversitätsforschung, Universität Wien, Rennweg 14, A-1030 Wien, E-Mail: anton.weber@univie.ac.at 